\title{
Survey on Communication and Networks for Autonomous Marine Systems
}

\author{
Artur Zolich $^{1}$ (D) - David Palma ${ }^{2} \cdot$ Kimmo Kansanen $^{3} \cdot$ Kay Fjørtoft $^{4}$ - João Sousa ${ }^{5} \cdot$ Karl H. Johansson ${ }^{1,6}$. \\ Yuming Jiang ${ }^{2} \cdot$ Hefeng Dong ${ }^{3} \cdot$ Tor A. Johansen ${ }^{1}$
}

Received: 28 April 2017 / Accepted: 28 March 2018 / Published online: 21 April 2018

(C) The Author(s) 2018

\begin{abstract}
The rapid development of autonomous systems and Information and Communications Technologies (ICT) create new opportunities for maritime activities. Existing autonomous systems are becoming more powerful and utilise the capabilities of several types of devices such as Autonomous Underwater Vehicles (AUVs), Unmanned Surface Vehicles (USVs) sometimes referred as Autonomous Surface Vehicles (ASVs) -, Unmanned Aerial Vehicles (UAVs), moored and drifting systems and, recently emerging, autonomous vessels. Their importance in providing new services in maritime environments is undeniable and the opportunity for coordinated and interconnected operations is clear. However, continuous wide integration of various technologies in maritime environments still faces many challenges. Operations may take place in remote locations, so that dependence on third-party infrastructures such as satellite communication or terrestrial communication systems must be expected. The reliability, performance, availability, and cost of such systems are important issues that need to be tackled. This work reviews the major advancements on state-of-the-art autonomous maritime vehicles and systems, which are used in several different scenarios, from scientific research to transportation. Moreover, the paper highlights how available technologies can be composed in order to efficiently and effectively operate in maritime environments. Highlights of the trade-off between autonomy and communication requirements are provided and followed by an overview of promising communication and networking technologies that could encourage the integration of autonomous systems in maritime scenarios.
\end{abstract}

Keywords Marine technology - Autonomous vehicles - Oceanographic techniques - Communication systems · Computer networks

Artur Zolich

artur.zolich@itk.ntnu.no

1 Center for Autonomous Marine Operations and Systems (NTNU-AMOS), Department of Engineering Cybernetics, Norwegian University of Science and Technology, Trondheim, NTNU, Trondheim, Norway

2 Department of Information Security and Communication Technology, NTNU, Trondheim, Norway

3 Department of Electronic Systems, NTNU, Trondheim, Norway

4 SINTEF Ocean, Trondheim, Norway

5 Department of Electrical and Computer Engineering, Porto University, Porto, Portugal

6 School of Electrical Engineering, KTH Royal Institute of Technology, Stockholm, Sweden

\section{Introduction}

Oceans cover more than $70 \%$ of the Earth's surface, being closely tied with life on Earth and climate changes. In addition to the direct impact on the Earth's biosphere [1], the oceans are crucial for freight transportation among other sectors of economic value such as fishing, petroleum, minerals and tourism [2].

Various types of manned and unmanned vehicles, as well as infrastructures such as oil platforms, fish farms, buoys and sensor systems, rely on various Information and Communications Technologies (ICT) and currently conduct operations in oceans and seas across the world. Despite the rapid development of ICT and autonomous systems in specific scenarios, their continuous wide integration in maritime environments still faces many challenges. 
This work analyses current challenges and opportunities for autonomous maritime operations and their strict dependence on networking and communication technologies. In particular, the following contributions are provided:

1. Review of state-of-the-art autonomous marine systems and scenarios

2. Characterisation of applications for marine autonomous systems

3. Classification of unmanned vehicles and target operations

4. Overview of existing and future communication and networking technologies

The use of unmanned vehicles has allowed higher-levels of precision and accuracy in many research expeditions [3, 4], as well as increased cost efficiency, when compared against typical research expeditions. This approach is particularly relevant in challenging or hazardous environments, and if real-time data exchange is required for conducting the research process [5]. Additionally, the cooperation and integration of unmanned vehicles with sensors can be used to improve the data-acquisition process and overall performance of the mission [5-8]. In fact, heterogeneous remote and in-situ sensing systems for maritime environments are envisioned in the near future [9-12]. For example, new transportation routes such as the Europe to Asia link through the Arctic will become more widely used [13] and, as with existing routes, several threats must be addressed such as drifting icebergs. This leads to the need of new systems capable of supporting ships in their navigation, complementing existing sensors and increasing robustness, precision and accuracy [4].

The impact of ICT and robotics in oceanography, and other maritime affairs, is reflected in the increasing demand for higher data-rates, data integrity, real-time communications and robustness. Nonetheless, the control and monitoring of these systems and the acquisition of research-data are challenged by the quality of data communications. In maritime scenarios, the lack of infrastructures considerably limits the access to telecommunication technologies so the use of satellite systems, or other long-range and low-bitrate communication systems, is the prevalent solution.

Currently, voice and similar low data-rate communications are the existing alternatives for maritime communication systems. For example, the Global Maritime Distress and Safety System (GMDSS) uses Digital Selective Calling (DSC) to send pre-programmed digital messages through standard maritime radios such as VHF. A different technique is employed by the Automatic Identification System (AIS), which introduces information about position, speed and heading, increasing Situational Awareness (SA) and, though limited to this information, preventing collisions. Regarding scientific data, very few communication options are available. For instance, satellite links such as Inmarsat and Iridium can allow global connectivity but have the drawback of being costly (financially and in energy efficiency), and provide only limited bandwidth. The providers are working with new systems, such as Iridium Next, a Low Earth Orbit (LEO) system, which should be available in $2018^{1}$. Additionally, alternative solutions resorting to High Elliptical Orbit (HEO) satellites covering Pan Arctic areas are also being considered [14].

Long-duration scientific missions often require manned support to retrieve scientific equipment after a given period of time. That can particularly happen for research purposes where a single sensor may generate megabytes of data per minute (e.g. sonar data [15]). In some cases such retrieval mission can be more expensive then the value of the sensor itself. That could change if affordable, fast and energy efficient way of communication with remote areas were available.

This lack of appropriate and widely available data-links restrains scientists in what can be done. For example, adapting the data-acquisition process according to received samples or environmental condition may be desirable, as well as combining sensors and actuators [16], which is mostly disregarded today. Moreover, the planning of manned missions for deploying and collecting sensors is extremely complex and costly, raising considerable crewsafety concerns, in particular when missions are far from shore.

The coordination of heterogeneous systems introduces the possibility of creating a communication network between vehicles. By handling these systems appropriately, cooperation can be promoted between different data producers and consumers without compromising operations, benefiting all involved actors. Nonetheless, this reality is yet to be fully exploited, and the used technologies depend mostly on what maritime actors acquire for their specific needs and vehicles, disregarding an interoperable networking opportunity. In fact, off-the-shelf equipment for communication purposes is typically employed in these scenarios [17], and there is a need for common standards and interfaces to interconnect such systems.

This paper reviews current trends, challenges and upcoming opportunities to enable the next-generation of coordinated autonomous systems for maritime environments, considering not only the use of unmanned vehicles but also communication technologies, standards and protocols capable of creating heterogeneous networks of maritime systems. An overview of state-of-the-art scenarios and operations is presented in Section 2, where various unmanned vehicles, with distinct levels of autonomy, are coordinated with other vehicles and infrastructures in real maritime

\footnotetext{
${ }^{1}$ https://www.iridium.com/network/iridiumnext
} 
operations. Section 3 presents a thorough review of marine autonomous systems, including details about different types of challenges and capabilities. Networking and communication technologies as enabling technologies for such systems are presented in Section 4, where the trade-off between fully autonomous and remotely operated missions is highlighted. Section 5 discusses technological enablers for maritime communication and networking. Finally, conclusions are given in Section 6.

\section{Background and Motivation}

Following the success of remotely operated vehicles, marine autonomous systems start playing an important role in sea operations and research. There is a number of systems available, tailored for different tasks and operations, having various capabilities and performance. The marine environment is harsh and demanding. Strong, gusting winds, followed by powerful waves can easily influence vehicles controlability, or damage their structures. For the autonomous systems that require vessel support, even deployment and retrieval can be a challenge.

There is a wide range of tasks where autonomous systems can be used in order to reduce crew risk, speed-up operations, or reduce their cost. However, such operations need matured and reliable technologies requiring minimum attention and maintenance from the crew, as every intervention is costly, takes significant amount of time and involves an increased risk.

One of the benefits of systems getting increased autonomy is reduction in number of crew members involved in their operation. Crew members can move their attention from controlling every single step of the vehicles into focusing on the high-level goals. The human role in unmanned systems operations has evolved over time. It started with direct control of the vehicles and is changing now into mission management and supervision. Additionally, if sufficient communication infrastructure is provided there is less need for the crew to be in the vicinity of the autonomous vehicles. The increasing level of autonomy allows to limit number of people on a ship, in the air, or at sea, and place them in a safe, and comfortable environment onshore. This allows the establishment of operations in hazardous areas, or in conditions that can be uncomfortable to the crew, e.g. longendurance operations where human operators' fatigue is a challenge. One example can be underwater intervention works, which normally would require highly-skilled divers, and that in the future can be executed by underwater vehicles with human operators in a control room [18]. Moreover, it creates new possibilities for cooperation in scientific explorations. With higher autonomy and therefore easier to operate vehicles, units scientists can focus more on high-level goals (i.e. scientific data collection, and its on-line interpretation), rather then low-level technicalities of controlling them. In addition more autonomous units should be easier to integrated into systems of various types of vehicles which can provide broader and more detailed picture of investigated phenomenon. Such on-line operation should support near real-time data acquisition and sharing, in order to monitor the progress of operations, enabling a quick reaction to unexpected events and more efficient use of resources. However, outside the coastal areas there is very limited access to a communication infrastructure other than satellites. Furthermore, in polar regions even satellite coverage is limited. In order to allow easier access to information in remote areas communication channels, protocols, and technologies should be standardised.

Current state-of-the-art operations are quite diverse. In this paper the operations involving autonomous vehicles are divided into three categories. The first one is heterogeneous robotic operations. These activities involve various types of autonomous and robotics vehicles, such as Autonomous Underwater Vehicles (AUVs), Unmanned Surface Vehicles (USVs) - sometimes referred as Autonomous Surface Vehicles (ASVs), and Unmanned Aerial Vehicles (UAVs). The idea behind these operations is to exploit specific capabilities of every vehicle to complete the high-level mission goals. Still, mostly because of limited endurance and range of systems, such operations are usually performed in the vicinity of a coastal infrastructure or a support vessel, and are limited in time.

The second group includes long term, remote activities, usually performed by moored and quasi-static systems. Buoys and floating platforms are typically used to measure environmental data. These devices are designed with a special attention to long lifetime, high reliability and durability in expected sea conditions. Due to very limited access to the communication and power infrastructure, the devices face strong constraints on the amount of information they can generate and transfer to the user.

The third type of marine activities, which are currently emerging, are unmanned ships and autonomous shipping operations. Unmanned ships that can navigate seas and deliver goods and passengers to their harbour of destination are being used and getting more attention in long-term industrial strategies.

This section provides more details on practical examples for each group of operations.

\subsection{Heterogeneous \& Robotic Operations}

Heterogeneous robotic operations in maritime environment are being demonstrated more and more often, typically resulting from a joint effort between multiple institutions and interdisciplinary fields of research. An example of such 
Fig. 1 Heterogeneous operations example, Mola-mola tracking interconnections. Adapted from [7]

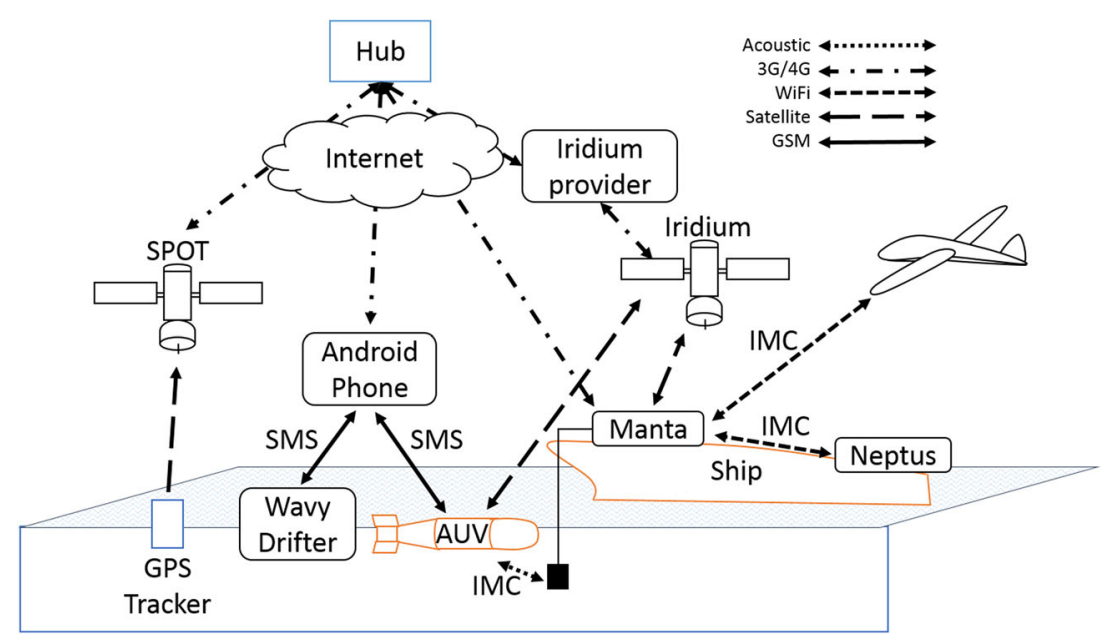

an operation was the Sunfish Tracking experiment ${ }^{2}$ in which a UAV and AUV, as well as a USV and manned vessel, were combined to track Mola-mola fish [7, 19]. That operation required continuous monitoring from several sensors, from cameras to sonars, in order to allow biologists to better understand behaviours and the environment.

The created system took advantage of various communication technologies presented in the Fig. 1. Inter-vehicle communication was realised using $\mathrm{WiFi}$, satellite, cellular and acoustic links. The central data-element of the system called a Hub - collected data from the sensors and provided it to the users and vehicles using satellite and cellular access to the Internet.

The Mola-mola fish are known for staying at the surface, from time to time, in order to warm up through the sun's radiation. This behaviour allowed to track a few specimens, while at the surface, by tagging them with a custom-made device, equipped with a Global Navigation Satellite System (GNSS) receiver and a satellite transmitter.

Even though the used device provided information about the fish's position, their next resurfacing time and position is unknown, and therefore the experiment has significant dynamics when trying to gather all the required data. This is one of the reasons why several types of vehicles, monitored and coordinated from a control centre, were used during the operation. High- and low-level control over the vehicles and the entire system was conducted using the LSTS Toolchain [20]. The unmanned vehicles were used to gather a wide set of data about the environment where Mola-mola fish live.

UAVs, AUVs, and USVs are characterised by very different capabilities when considering the spatial domain, time of operation and deployment, and sensors. In the Mola-mola tracking and monitoring experiment, whenever a tagged fish re-surfaced and the tag's GNSS acquired

\footnotetext{
${ }^{2}$ http://sunfish.lsts.pt/en
}

satellite signal, its position was sent to an Internet server via the satellite link. This allowed the researchers, in the control centre, to trigger appropriate actions. For example, the USV, constantly operating in the region, was commanded to navigate to the position of a fish at the surface, while registering required water parameters. Finally, if the fish's position was within the range of the available UAV, another team of researchers was prepared to launch the aeroplane to track and capture video footage of the surfacing animal.

The UAV used in the experiment was based on a Skywalker X8 platform, equipped with a High-Definition (HD) camera and capable of a flight time of 60 minutes. This unit has a range of 8 to $10 \mathrm{~km}$, with a cruise speed of $18 \mathrm{~m} / \mathrm{s}$.

The AUV used in the experiment was a Light AUV (LAUV), capable of operating under the water for up to 8 hours, reaching a speed of $3 \mathrm{kn}$, and a depth of 100 meters. It was also equipped with a Conductivity Temperature and Depth (CTD) sensor, a fluorometer and an HD camera. Side-scan sonars and multibeam echo sounders could also be mounted if required by the researchers for other measurements.

The USV was a WaveGlider, which is a boat powered by waves and capable of moving with speeds between 0.5 and $1.6 \mathrm{kn}$, depending on the sea conditions. It was equipped with an Acoustic Doppler Current Profiler (ADCP), a CTD and a weather station. Passive propulsion and solar energy harvesting technology allows this vehicle to perform very extensive missions in time (years), limited only by maintenance needs.

\subsection{Moored and Quasi-Static Operations}

There is a significant number of moored or drifting nodes deployed all over the world. The Global Ocean Network statistics list 125518 moored systems active between 2002 and 2016 [21]. Moored and quasi-static 
systems can be seen as unmanned nodes capable of carrying several different sensors, enabling them with a physical infrastructure, processing and communication capabilities. They are characterised by their sensors and lifetime expectation, which commonly spans over at least one year. This requires wise and efficient energy management, such as task planning and communication scheduling. The communication technologies may rely on satellite links, but may also require additional interactions to collect the vast amount of data generated by different nodes.

The lifetime and durability are two of the key aspects of moored and quasi-static systems. Such systems are used for long term deployments where continuous observation is important. The way sensors collect data is unique to every device family. Limited power and communication resources reduce the amount of data retrieved, and deployment endurance may be favoured over the sampling duration and frequency. Many devices are equipped with energy harvesting modules, which can use solar, wave or wind energy to support their power systems. However, in certain locations these technologies may fail, e.g. in rough seas or during Arctic night. In addition, energy converters increase the size, cost and complexity of the devices.

Floating nodes are often a support for underwater sensor collecting measurements such as salinity, temperature, density, and light level. Moreover, information about drifting nodes position is used to model sea currents [22]. Each device is made with a specific lifetime in mind. Similarly, the sampling frequency and duration is selected by the scientists who are familiar with processes they observe.

Several examples of moored and quasi-statics operations that vary in terms of scale, scope and method can be provided. An $\mathrm{Argo}^{3}$ array is part of the Global Climate Observing System/Global Ocean Observing System GCOS / GOOS. The ARGO observation systems consist of approx. 3800 free-drifting nodes that measure temperature and salinity of the upper $2000 \mathrm{~m}$ of the ice-free ocean. Motion of the nodes is tracked providing information about the current. Data are collected using satellite communication. Two satellite systems are being used, Systéme Argos and Iridium. In case of Systéme Argos the node need to spend between 6 and 12 hours at the surface to transfer its data and to measure its position. Maximum positioning accuracy is approx. 100 meters, and depends on satellites number and geometry. When the Iridium system is used, position is acquired from Global Positioning System (GPS). In 2015, 65\% of floats were deployed with Iridium and $35 \%$ with Argos [22].

Another example are arrays of acoustic receivers which are used to track tagged fish in the area of interest. In this

\footnotetext{
${ }^{3}$ http://www.argo.ucsd.edu/
}

case the nodes usually consist of passive acoustic receivers, that records acoustic tag transmissions. Because the receivers cannot transmit the information further, data need to be collected manually [23]. Due to a significant number of nodes, the retrieval operation consumes considerable time and resources. In addition, the data collection campaign may need to be repeated up to 4 times a year and require professional team, e.g. scuba divers. The amount of data collected during the entire process depends on fish activity and fish tag transmission settings. For example, each receiver can collect up to 1500000 entries, which is roughly a dozen MBs. Total amount of data collected depends on the number of receivers, however not necessarily linearly, as fish activity is not uniformly distributed.

In some cases the data from array may be collected by unmanned vehicles. During one campaign of a WaveGlider, a passively powered USV, was used to collect data from 184 underwater tracking systems, distributed over $205 \mathrm{~km}$ of distance [24].

In another scenario, a technology demonstration was built, where underwater receivers were supported by a surface unit [25]. The surface part was equipped with Wireless Sensor Network (WSN) nodes, which allowed to download the data from the underwater sensors. The mesh-type WSN network used an $868 \mathrm{MHz}$ Industrial, Scientific and Medical (ISM) communication link. A small commercial off-the-shelf multi-rotor was used as a relay node to demonstrate data relay mechanism. Tests resulted in a few $\mathrm{KB} / \mathrm{s}$ transmission speed, which was considered sufficient for the scenario.

Another type of moored operation is being conducted by the ArcticABC project, ${ }^{4}$ which involves extensive data collection from several different and complex sensors, including Underwater Hyperspectral Imager (UHI) units, HD cameras and Acoustic Zooplankton Fish Profiler (AZFP) units [26].

The system can be deployed in locations where no suitable satellite coverage, or other typical communication systems, are available and where physical access is very limited. In order to circumvent these limitations and high-bitrate requirements, UAVs are being considered for data collection, in which the moored nodes will gather information about the Arctic environment [27].

In the Arctic $\mathrm{ABC}$ project, a group of biology researchers and engineers is building a set of ice-surface nodes in order to record environmental observations. 6 types of Icetethered Platform cluster for Optical, Physical and Ecological sensors (ICE-POPEs) are being developed:

1. Ice parameters monitor $(\mathrm{P}-1)$

2. Underwater light measuring node $(\mathrm{P}-2)$

3. Acoustic Zooplankton Fish Profiler node (P-3)

\footnotetext{
$\overline{{ }^{4} \text { http://www.mare-incognitum.no/index.php/arcticabc }}$
} 
4. Underwater Hyperspectral Imager node (P-4, Fig. 2)

5. Weather station (P-5)

6. Archival unit (P-6)

ICE-POPEs P-1, P-2 and P-5 generate few KB of data per day, where measurements and control data will be transmitted entirely via a satellite link. P-3 and P-4 carry instruments that generate a significant amount of data. Specifically, P-3 is expected to generate tens of MB of data per day, while $\mathrm{P}$ 4 is expected to generate 1 GB of data per day. P- 6 is going to provide a backup storage for P-3 and P-4 and, depending on the number and type of deployed ICE-POPEs, P-6 is expected to collect dozens of GB of data per week.

There are 3 ways of accessing data on P-3, P-4, and P6 units. The primary communication channel is an Iridium Short Burst Data (SBD) Service modem. The SBD message arrives at a microcontroller and informs it about a schedule of power cycles for every component of the unit: the main Single Board Personal Computer (SBPC), a highspeed radio, an Iridium dial-up modem, and the sensors power. At a predefined time, the microcontroller sends back a message that contains house keeping information, e.g. battery level, status of the device and its components. When the microcontroller powers-up all the components, the main SBPC of each unit can be accessed using the Iridium dial-up

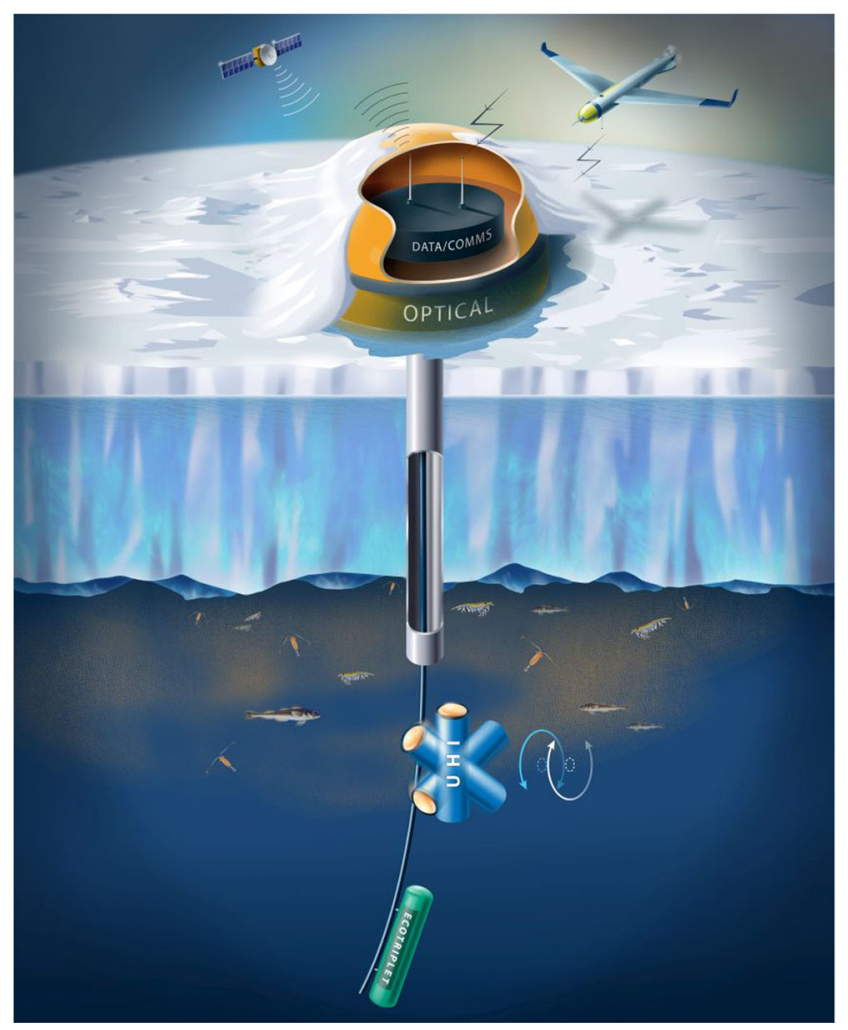

Fig. 2 Moored operations example, an ArcticABC Underwater Hyperspectral Imager node P-4 (Copyright Bjarne Stenberg/NTNU) modem. Via the dial-up channel a configuration change can be applied, and some parts of recorded data retrieved from any location on the world. The main data retrieval channel is a high-rate radio operating in the ISM band. Depending on the region, a $900 \mathrm{MHz}$ or $2.4 \mathrm{GHz}$ technology is considered. Because the high-speed radio has a limited range of a few kilometres, the user or a data-mule need to be present in the vicinity of the node in order to collect the data stored onboard the unit. The initial idea is that data will be gathered from a vessel that accommodates researchers near the area of deployment. The second option is the use of long-range UAVs that flies to the area of deployment and retrieve the data. The third possibility is physical retrieval of the P-6.

The ice-layer behaviour may cause units P-3 and P-4 to not be retrievable. For that reason, during the deployment the units P-3, P-4 and P-6 should regularly synchronise data stored in their internal memory using the high-speed radio link. The P-6 design will make it less complex to detach from ice, so it can be collected by a manned expedition that arrives on an ice-breaker or by an aerial vehicle. ${ }^{5}$

\subsection{Unmanned Ships and Autonomous Shipping}

The concept of unmanned ships offers potential advantages in regard to vessel design and construction as well as reduction in operating costs such as fuel, labour, and environmental footprint relative to traditional manned ships. Recently there has been significant interest in developing such systems. Applications include short sea cargo shipping [29], longer distance freight [30], and ferries [31]. At the present state of development, it is still considered a requirement that a human operator needs to be responsible and in command of the unmanned ship. That typically leads to the critical requirement of a communication link between the ship and an onshore operator centre, where piracy are primary concerns in addition to safe manoeuvring, situation awareness and fault tolerance [32, 33].

Autonomous operation of a surface vehicle requires that guidance, navigation and control are performed with high reliability, fault-tolerance, and safety. It includes realtime perception of the ship's surroundings in order to avoid grounding and collision with other ships, vessels, people, marine mammals or other obstacles that may be encountered. In order to be able to detect the wide range of potential obstacles and provide automatic collision avoidance and situation awareness, on-board sensors such as radar, Light Detection and Ranging (LIDAR), and camera can be used to scan the environment of the ship [35-38]. ${ }^{5}$ For the time being such scenario has limited feasibility but that may
change in the future [28] 
An E-navigation global strategy is intended to meet present and future user needs through harmonisation of marine navigation systems and supporting shore services. Future user needs must also focus on autonomous operations, as well as how the manned vessels are interacting with unmanned vessels, and the role of the traffic centres operating unmanned vessels. Regulations and standardisation are also issues of concern. The International Maritime Organisation (IMO) defines E-navigation as "the harmonised collection, integration, exchange, presentation and analysis of marine information on board and ashore by electronic means to enhance berth to berth navigation and related services for safety and security at sea and protection of the marine environment." In November 2014 the E-navigation Strategy Implementation Plan (SIP) was approved, where one of the scopes concerned effective and robust communication methods for maritime operations.

Rules for ship collision avoidance are given by the Convention on the International Regulations for Preventing Collisions at Sea (COLREGS), by the IMO [39]. Whilst COLREGS were made for ships operated by a crew, their key elements are also applicable for automatic collision avoidance systems, either as decision support systems for the crew or in autonomously or remotely operated unmanned ships. In an autonomous system implementation, COLREGS implicitly impose requirements on the information that must be provided by sensor systems, and the correct actions that should occur in hazardous situations. Ships (and autonomous ships in particular) are expected to carry an Automatic Identification System (AIS) broadcasting radio signals containing position, velocity, and other information about the ship, that can be received by other ships and authorities. COLREGS also demand communication by light and sound signals, which one might expect will be extended by means of radio broadcast protocols in the future to support autonomous and unmanned ship operations.

A manned vessel today have from 400 to several thousand sensors that in one way or another are reporting data or are used in an operation. The number of sensors will not decrease when the vessel will be autonomous, and we also see the need of reporting some of the data to a shore-based control centre such that the vessels status is under control. Rolls Royce have announced that they are about to start developing a remote control centre for fleet management, also with regards to unmanned vessel operation [40]. Remote control and autonomy are priorities. This results in a high requirement to the communication infrastructure, as well as to security and integrity of the data to be exchanged between vessel and a shore based centre.

Since 2015 Rolls-Royce has led a joint industryacademia research project called Advanced Autonomous Waterborne Applications Initiative (AAWA) [41], presenting a concept of a fleet of unmanned ships, controlled by a limited crew from an on-shore control centre. This concept includes the vision of a futuristic bridge, equipped with state-of-the-art automation technologies, believed to be part of modern ships by the year 2025 [42]. Currently, series of tests are planned using a 65 meters double-ended ferry, the Stella, in Finland (Fig. 3). These tests will answer the question how to combine existing communication technologies, and the use of unmanned vehicles, in a way that enables autonomous ship control. Similar initiatives of autonomous ships were also presented by MUNIN - Maritime Unmanned Navigation through Intelligence in Networks where vessels are primarily guided by an automated onboard decision system and controlled from the shore [43].
Fig. 3 Autonomous shipping example the Stella ferry [34]

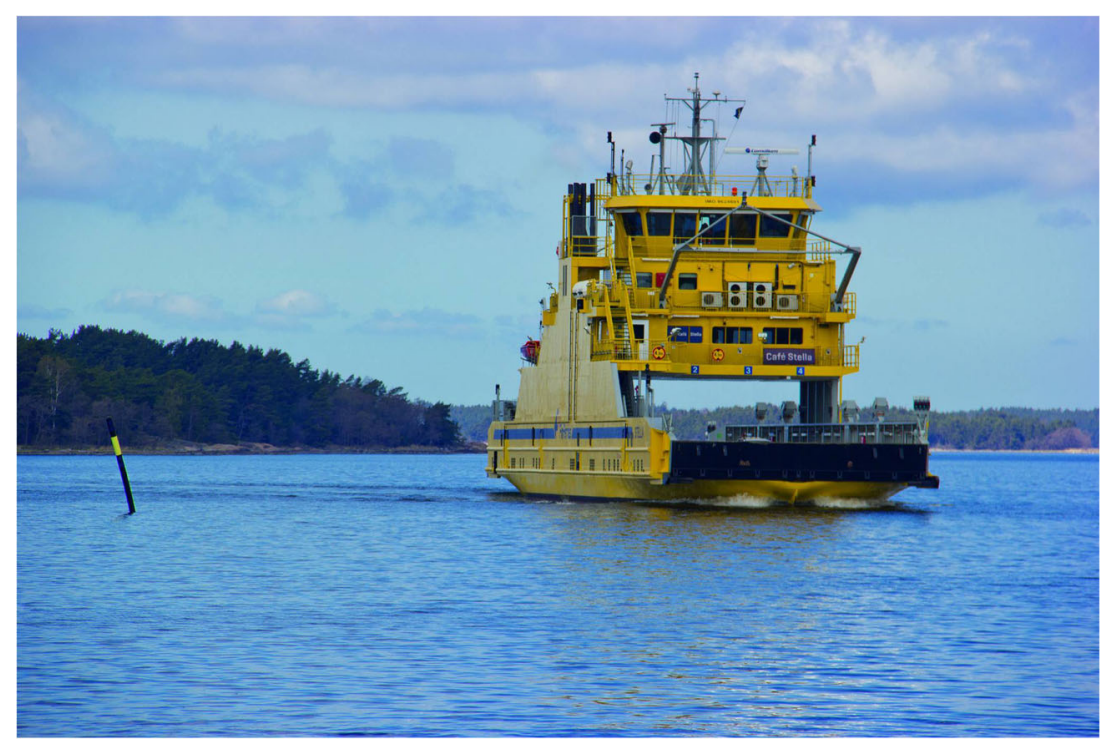




\section{Applications of Marine Autonomous Systems}

Autonomous systems in the marine domain range from moored buoys, drifters, AUVs, USVs, UAVs and conventional manned vehicles, as well as more persistent infrastructure such as satellites in space, seabed transponders, and coastal and onshore communication assets. An illustration is provided in Fig. 4. Many systems utilise several of these assets, for activities ranging from military operations, oceanographic research, to offshore marine operations with industrial and shipping companies. Common to all vehicle types is that they can be operated manually (remote control), automatically according to pre-programmed paths or trajectories, or autonomously by on-board planning and re-planning.

This section provides a general perspective on autonomous vehicles and types of operations that are present in the literature.

\subsection{Missions and Objectives}

Maritime mapping, monitoring, surveillance and observations have diverse objectives that largely influence the area under study. For instance, observing a fish farm may require the monitoring of no more than a few kilometres. However, obtaining migration data of large animals that travel large distances, such as whales, requires a global-scale observation. Bearing such diversity in mind, this work considers four scales of operation.

\section{Small scale:}

Operations concerning the coordination of self-contained system for remote-sensing within a few kilometres;

\section{Medium scale:}

Sensing missions that can cover up to tens of kilometres;

\section{Large scale:}

Initiatives that may include the cooperation of different teams and infrastructures, accounting for hundreds of kilometres;

\section{Global scale:}

Operations without fixed boundaries, typically involving several actors.

The perception of scale in a mission may result from the perspective of a single-user, or a research-team, in which interoperability is foreseen between vehicles belonging to that action. Nonetheless, the use of standard protocols and interfaces can allow a researcher to embark on a global mission even with a limited number of vehicles. For example, by solely deploying drifting nodes, a researcher may remotely access its sensors' data through several other vehicles operating around the world that are able to forward it.

In many cases operations need to be planned long time ahead, because of vessel availability, environmental phenomenon cycles (e.g. yearly storms) or budget. Robotic operations can involve a variety of vehicles with different performance and capabilities. Mission goals are tailored to fit capabilities of these vehicles. Although the main objectives are well defined, the mission plan usually need to adapt to circumstances on site.

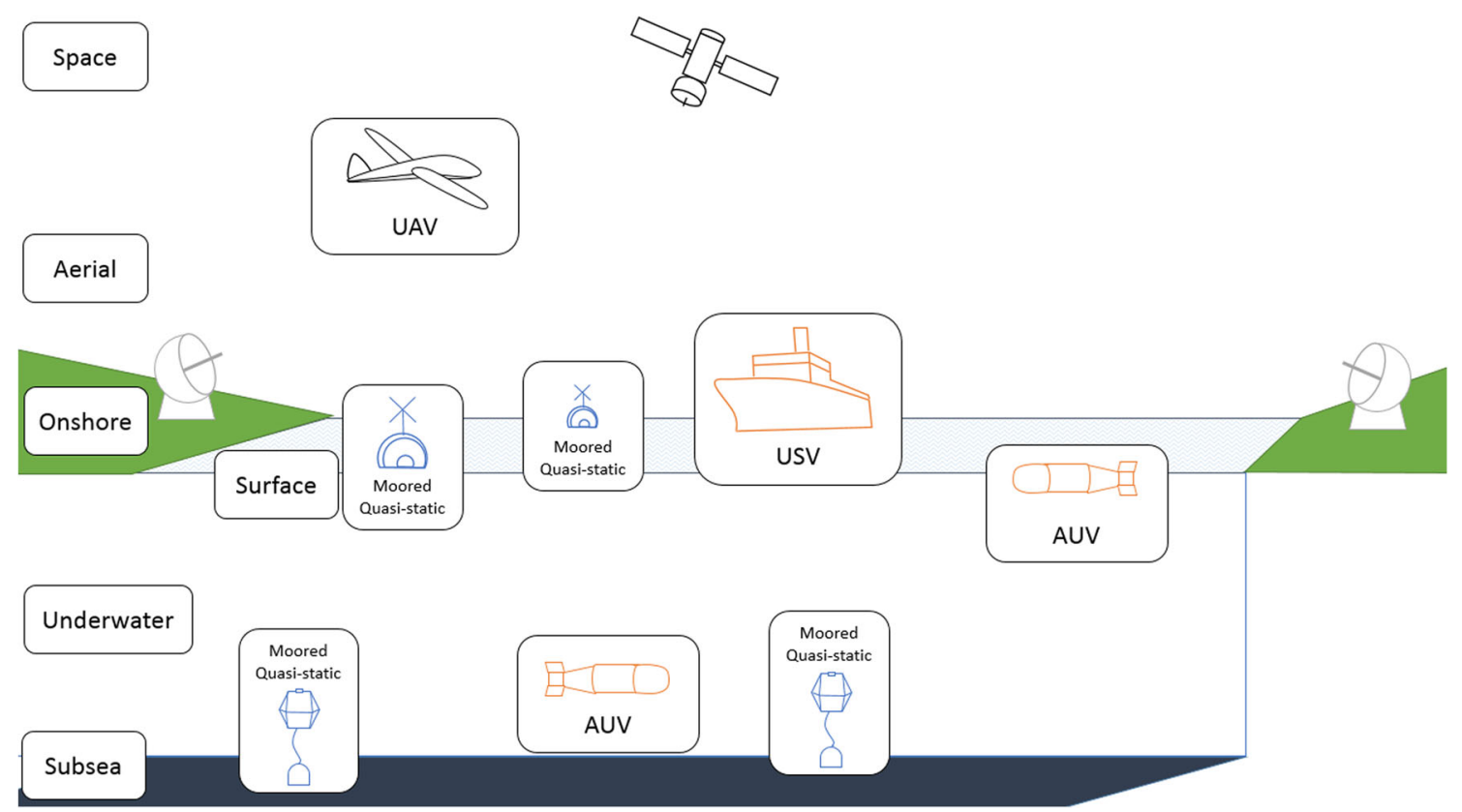

Fig. 4 Example of the types of typical nodes (mobile and fixed) that may be present in communication network supporting autonomous marine systems and operations 
The global cooperation between multiple vehicles and infrastructures may result from agreements between different parties (e.g. multiple research teams), similarly to the creation of the Internet. This global perspective can be enabled by use of standard protocols and interfaces, with some added cost for forwarding nodes. For some vehicles this cost may be too high and therefore they will not act as forwarding nodes, but for others it might be negligible. For example, existing infrastructures and large vessels typically have enough resources so that they can act as data mules to piggy-back research data (i.e. relay or forward data).

Planning and execution of operations that involve different types of unmanned vehicles are possible thanks to a compatible communication and control architecture. One of the key components of such architecture is a C2 (Command and Control) software [20], or its variations, which provides Situation Awareness to the user, together with tools to control the vehicles tasks. Often, autonomous vehicles execute itineraries defined by the human operator. However, when assets works under a common C2 system a new mission planning mechanism can be applied and mission-plan definition can be automated [7]. In many cases, validating that a mission plan was successful is possible only after post-processing of collected data. Higher level of autonomy on-board the vehicles, enables them to adapt their trajectories and tasks to maximise mission outcome on-line.

Using unmanned vessels for transportation of goods around the world introduces a new paradigm of safer and more economically viable transportation. These unmanned ships have a significantly increased autonomy when compared to smaller unmanned vehicles, but still require environmental scanning in order to avoid obstacles and debris, among other threats. In addition to using shipmounted sensors, this environmental scanning may be possible by resorting, for instance to UAVs, that can perform scouting surveys in front of the bigger ships, using a multitude of different sensors, [44]. The use of compact, vertical take-off, unmanned aerial vehicles for surveillance of the ships structures is in fact one of the presented features in the Rolls-Royce vision [31].

The use of unmanned vehicles may serve as monitoring equipment that needs to be operated from the larger ships' infrastructure. For this reason, Vertical Take-off and Landing (VTOL) UAVs may be favourable, allowing for a smoother operation. These UAVs may be equipped with several imaging sensors or simply collect or relay information from moored systems, as presented before, that may still not be available. Other smaller scale vehicles, such as AUVs, can be used to perform complementary environmental sensing such as underwater hull checks, or even detection of underwater obstacles before the larger scale ships are even close to them [45]. This improves not only the autonomous navigation of the ships but it also reduces maintenance costs and security threats, which cannot be provided by other systems such as current satellite monitoring solutions [46].

Moored or quasi-static systems with similar characteristics typically collect amounts of data suitable to be transmitted over the satellite network for an extensive period of time [22, 26, 47]. In some cases, due to the complexity of the instruments that may generate vast amount of data in a short period of time, or due to design limitations, satellite communication cannot be applied [26]. In that case data needs to be manually collected, often a few times a year [23]. Such operations require significant crew involvement, often including costly vessel time and scuba-divers. Manually picking up nodes/data does not scale well, as there are many harsh scenarios where environmental hazards do not encourage or permit human presence. To make things worse, in several situations there is no near-real-time access to the nodes' data and status, and therefore researchers have no insight into the situation and cannot react to changes or failures. Bearing this in mind, near-real-time access to data would allow researchers not only to access relevant environmental information faster, but also to reduce the operational costs and the risk of losing data [48]. These interactions can result from the use of additional unmanned vehicles such as UAVs, USVs or AUVs.

\subsection{Autonomous and Remotely Operated Vehicles}

As previously seen in Section 2, the coordinated use of multiple heterogeneous unmanned vehicles for remotesensing can be beneficial for several reasons. Not only can different vehicles be used to acquire data in different mediums (e.g. underwater, surface and aerial), but they can also improve the overall communication performance. The area of operation, however, must also be taken into consideration when selecting remote unmanned vehicles that may enhance data acquisition.

The Table 1 presents examples of autonomous vehicles utilisation in various scales of operations found in the literature.

\subsubsection{Autonomous Underwater Vehicles (AUVs)}

AUVs have found wide-spread use for science, mapping, industrial inspection and defence/security applications, e.g. $[58,59]$. They typically have payload sensors for surveillance, remote sensing (side scan sonar, camera systems) and measurement of physical and chemical properties of the ocean. While submerged, both communication and inertial navigation aiding are primarily restricted to hydro-acoustic systems, which have limitations related to bandwidth, cost and power consumption. Although not ideal from an 
Table 1 Autonomous vehicles used at different operations scale present in the literature for Maritime Environments

\begin{tabular}{|c|c|c|c|c|c|c|c|c|c|c|}
\hline & \multicolumn{2}{|l|}{ UAV } & \multicolumn{3}{|l|}{ AUV } & \multicolumn{3}{|l|}{ USV } & \multicolumn{2}{|c|}{ Stationary } \\
\hline & $\begin{array}{l}\text { Smaller } \\
(<25 \mathrm{~kg})\end{array}$ & $\begin{array}{l}\text { Larger } \\
(>25 \mathrm{~kg})\end{array}$ & LAUV & AUV & Gliders & $\begin{array}{l}\text { Renew. } \\
\text { Energy }\end{array}$ & Boats & Vessels & $\begin{array}{l}\text { Moored } \\
\text { buoys }\end{array}$ & $\begin{array}{l}\text { Drifting } \\
\text { buoys }\end{array}$ \\
\hline Small-scale (0-10 km) & {$[16,25]$} & & {$[7,19]$} & & & [49] & {$[50,51]$} & & {$[25]$} & {$[52,53]$} \\
\hline Medium-scale (10-100 km) & & {$[54]$} & & {$[8]$} & {$[55]$} & {$[19,56]$} & {$[8]$} & & & [19] \\
\hline Large-scale (100-1000 km) & & & & & & {$[24,56]$} & & & {$[26]$} & [19] \\
\hline Global-scale (>1000 km) & & & & & & & & {$[43,57]$} & {$[26,47]$} & \\
\hline
\end{tabular}

operational point of view, AUVs may be commanded to the surface in order to enable radio/satellite-based communication and navigation, which is still challenging since antennas may still be partly submerged due to ocean waves. Due to limited endurance (few hours to few days [60]) and limitations in communication under the water [61], tasks of AUVs are usually pre-programmed and data analysed after vehicle recovery. However, progress in autonomy mechanisms enables some vehicles to adjust its behaviour on-line during mission execution, in order to maximise user-defined outcome [62]. AUVs have also been utilised as data mules in similar scenarios, exploiting the use of short-range optical links for increased data transfers, as opposed to the typically used acoustic links [63].

A special case of an AUV is a Glider [60]. Gliders are long-endurance underwater vehicles, which can be equipped with a mechanical propulsion system, however main moving force is generated by relocation of centre-of-balance and buoyancy causing water to wash wings and therefore generate force that causes the vehicle to move forward [55]. These vehicles, designed for long range and long endurance operations, collect water column information during their dive. They transmit the collected data using a satellite communication link when they reach the surface afterwards. At the surface a modification or a new mission itinerary can be applied using the same satellite link.

\subsubsection{Unmanned Surface Vehicles}

USVs range from small platforms [64], to full-size ships [57]. USVs, similarly to all categories of unmanned vehicles, are used in multiple roles with various objectives. Small USVs have been widely researched for scientific missions (physical and biological oceanography), mapping and industrial survey $[50,56,65]$. There are vehicles dedicated to ice-berg monitoring [66], approaching dangerous waters glaciers [51], and environmentally-friendly operations on lakes [49]. Long-endurance, but low-speed, vehicles are commonly used for persistent surveys and environmental monitoring of selected area or traversing large distances [67]. Short-endurance, but fast, vehicles could be used for fast-response actions or patrolling [68, 69]. Specialised vessels are also used for high-risk military operations such as submarine hunting [57] and mine hunting [70]. USVs can also be used as carriers or support of other types of unmanned vehicles (e.g. UAVs [71-74], AUVs [75]) or act as communication relays between over-the-air/satellite communication and underwater vehicles/structures [8], or as data-mules [24].

\subsubsection{Unmanned Aerial Vehicles}

Unmanned Aerial Vehicles are commonly used in a wide range of tasks related to monitoring and surveys using various type of remote and in-situ sensing devices, such as optical cameras (RGB or hyper-spectral in the infrared or visible spectrum), laser scanners, radar and atmospheric sensors $[54,76]$. From a communications perspective they can operate as a mobile elevated antenna, creating a relay between nodes on the water surface and an operations centre [16, 25], or act as data-mules [77]. ${ }^{6}$ UAVs can also be utilised for carrying loads [78]. Some UAVs can be deployed and operated from small ships, while larger systems depend on onshore infrastructures for launch and recovery. UAVs are complementary to surface and underwater vehicles since they operate in the air at some altitude, and can thus observe and communicate from a different position and speed.

\section{Autonomy and Communication Trade-Off}

Taxonomies for the levels of autonomy in different types of systems have been presented in the literature by several authors [79-82]. For example, Autonomy Levels (AL) for Unmanned Marine Systems have been presented in [80]. These are:

- AL 0 Manual - No autonomous function. All action and decision-making performed manually, where humans

\footnotetext{
${ }^{6}$ The presented work is not related to the maritime environments, however it presents approach that could be used in one
} 
controls all actions (n.b. systems may have level of autonomy, with a Human in the loop).

- AL 1 On-board Decision Support - All actions taken by human operators, but decision support tools can present options or otherwise influence the actions chosen. Data is provided by systems on board.

- AL 2 On \& Off-board Decision Support - All actions taken by human operators, but decision support tools can present options or otherwise influence the actions chosen. Data may be provided by systems on or offboard.

- AL 3 'Active' Human in the loop - Decisions and actions are performed with human supervision. Data may be provided by systems on or off-board.

- AL 4 Human on the loop, Operator/ Supervisory Decisions and actions are performed autonomously with human supervision. High impact decisions are implemented in a way to give human operators the opportunity to intercede and override.

- AL 5 Fully autonomous - Rarely supervised operation where decisions are entirely made and actioned by the system.

- AL 6 Fully autonomous - Unsupervised operation where decisions are entirely made and actioned by the system during the mission.

A similar classification for the aerial vehicles can be found in [81]:

- 0 - Human operated - All activity within the system is the direct result of human-initiated control inputs. The system has no autonomous control of its environment, although it may have information-only responses to sensed data.

- $\quad 1$ - Human assisted - The system can perform activity in parallel with human input, acting to augment the ability of the human to perform the desired activity, but has no ability to act without accompanying human input. An example is automobile automatic transmission and anti-skid brakes.

- 2 - Human delegated - The system can perform limited control activity on a delegated basis. This level encompasses automatic flight controls, engine controls, and other low-level automation that must be activated or deactivated by a human input and act in mutual exclusion with human operation.

- 3 - Human supervised - The system can perform a wide variety of activities given top-level permissions or direction by a human. The system provides sufficient insight into its internal operations and behaviours that it can be understood by its human supervisor and appropriately redirected. The system does not have the capability to self-initiate behaviours that are not within the scope of its current directed tasks.
- 4 - Mixed initiative - Both the human and the system can initiate behaviours based on sensed data. The system can coordinate its behaviour with the human's behaviours both explicitly and implicitly. The human can understand the behaviours of the system in the same way that he understands his own behaviours. A variety of means are provided to regulate the authority of the system with respect to human operators.

- 5 - Fully autonomous -The system requires no human intervention to perform any of its designed activities across all planned ranges of environmental conditions.

The level of autonomy in a system is strongly correlated with the communication capabilities required by such system, as illustrated in Fig. 5. In general, high levels of autonomy means low communication requirements, while low levels of autonomy and automation will require more communication. In the extreme case of remote operation, the demands for capacity, latency and connectivity are very high. The lower left corner in the figure indicates an unfeasible situation, while the upper right corner is an ideal situation which enables higher performance and enhanced functionality beyond the minimum requirements.

Despite current advancements on autonomous operation, the control of large-scale unmanned ships, for example, depends on many factors such as the existing amount of surrounding environmental information. This may influence the levels of autonomy that can be achieved, and therefore the respective communication requirements. Depending on chosen or possible control mode, a manual remotecontrolled operation may require up to $4 \mathrm{Mbits} / \mathrm{sec}$ for live video, although significantly less bandwidth will typically be required in semi-autonomous or fully autonomous modes [32].

In addition to data needs related to the control of vehicles, other requirements and objectives must be considered when discussing communication performance. A taxonomy of typical data contents exchanged in a network of autonomous vehicles is as follows:

\section{Command and control (telemetry)}

Data includes commands in the form of goals, mission plans, parameters, low-level control commands from remote operator, payload commands, safety and emergency commands. Feedback data includes sensor measurements and alarm messages.

\section{Monitoring}

This may be the status of vehicle and payload systems, including communication systems. It may also include environmental data relevant to the control of vehicles.

\section{Payload data (mission)}

Several scenarios may include data collection as part of the mission. This can include a wide range of data, with 
Fig. 5 Levels of autonomy from [81], and how they relate to the requirements for communication

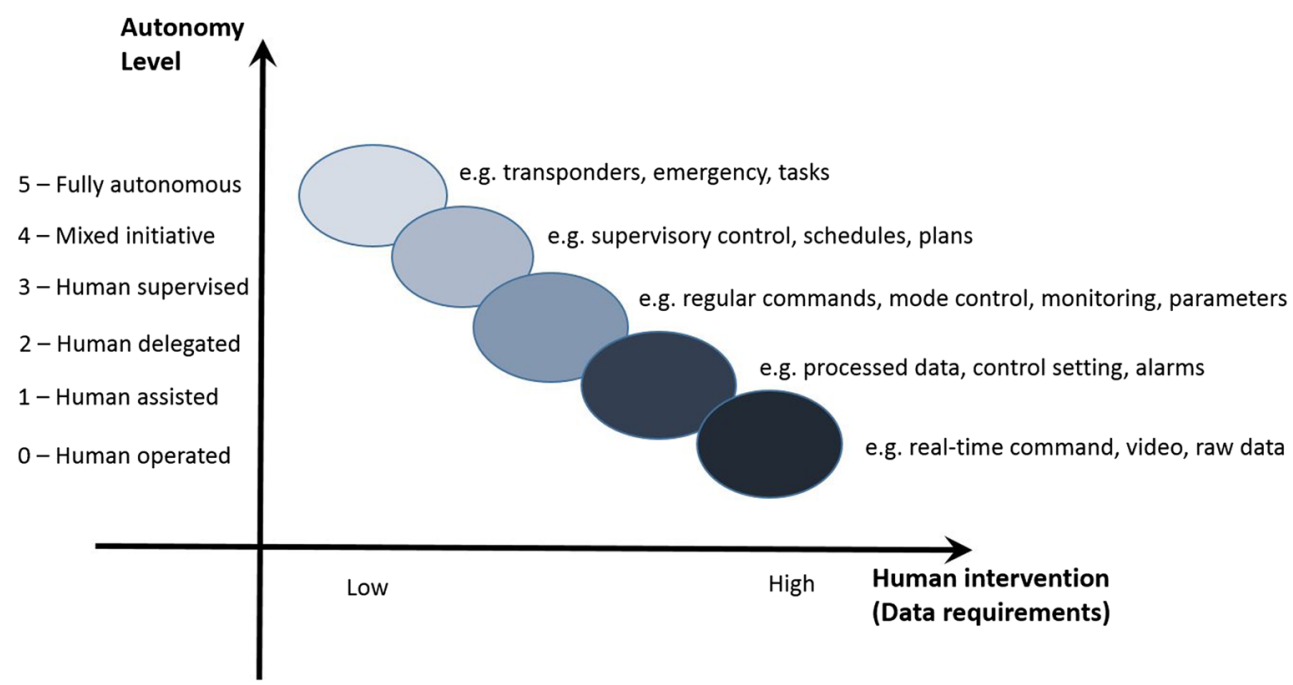

different requirements, from raw to processed data (e.g. compressed, aggregated or analysed data).

\section{Network Management}

Data exchanged to maintain a network of connected unmanned vehicles may also incur additional overheads that should not be disregarded. This may include the establishment of communication links or even relaying of data between vehicles.

An inherent characteristic of unmanned vehicles, closely related to communication requirements and performance, is mobility. It introduces possibly large distances between the nodes and the presence of different technologies for underwater and aerial communications. This implies that, in a heterogeneous network, existing assets that can operate at the ocean surface might have both hydro-acoustic and radio communication capabilities, including a bridge between the two technologies. However, unmanned systems can be small due to limitations in Size, Weight and Power (SWaP), which induces further limitations on communication. These can include the availability of continuous power or antenna sizes. Moreover, the communication between distant nodes at, or close to, the surface, is usually challenging due to a low antenna elevation, curvature of the Earth, and effects from ocean waves and surface reflections on the radio waves.

Controlled mobility of network nodes opens then possibility for communication- and networking-aware control of the position and attitude of the nodes. This can be exploited to optimise the communication performance jointly with other existing objectives and constraints. Nonetheless, some nodes are passive, being either fixed or drifting, and require the cooperation of other, more capable vehicles. In this sense, and in selected applications, the primary purpose of a UAV or USV could be relaying data over a communication network, where the vehicle can be positioned to optimise communication performance between devices [8, 16, 83]. Such an approach can be employed by resorting to models capable of predicting communication performance (e.g. path loss or attenuation) and defining the optimal position of autonomous aerial and surface vehicles [84, 85], for example in the acquisition of data from WSN nodes that are either moored or freely floating [86]. The modus operandi should take into account the vehicles' endurance and speed in order to be able to approach the sensing sites. After reaching the destination, the unmanned vehicle stays in the area in order to either retrieve or forward the available data. As mentioned in previous sections, energy efficiency in moored and quasi-static systems is of paramount importance. As such, the departure of unmanned vehicle from supporting vessels or other infrastructures such as docking stations, and its arrival to the site should take into account the scheduling of radio activation from the buoys side. By doing so, the available communication window between unmanned nodes is optimised and more data can be gathered.

\subsection{Challenges and Opportunities}

A wide range of communication technologies are available and can be considered for the unmanned and autonomous systems previously described. In fact, different High Frequency (HF) or Very High Frequency (VHF) radio technologies have been extensively used in the past for maritime communications. However, these are typically considered for manned communication systems (e.g. voice and Digital Selective Calling, DSC), not being suitable for new challenges and requirements introduced by the use of heterogeneous autonomous unmanned vehicles. Currently, existing challenges include not only the management, control and physical resources of the vehicles, but also the gathering and forwarding of large amounts of data generated by payload sensors. On the other hand, a unique opportunity 
for a coordinated control and operation of networks of underwater, aerial and surface vehicles is arising. This enables their complementary characteristics (operating space, speed, sensors, endurance, communication link, etc.) and extends the limits of operational flexibility and sensing capabilities [7, 69, 87]. Moreover, with the support of adhoc network structures vehicles are allowed to come and go, and contribute to relaying or transport of data. Examples of such opportunities include data collection in moored and quasi-static systems, which can produce an extensive amount of information every day, but that are typically limited by low-power and low-range communication. In this case, USVs or UAVs can be used for extending communication links and improve the overall lifetime of the system, see Fig. 6 .

In many unmanned applications (e.g. surveillance), communication requirements must be traded against the mobility requirements of the vehicle that are needed for positioning itself for the surveillance task. For example, in a search and rescue, or inspection task, there are waypoints or areas that the vehicle must survey. If this implies that communication link capacity is reduced or lost due to range or altitude, the impact of increased payload data latency and reduced payload data quality must be assessed. Using mechanisms such as delay-tolerant networking, the payload data can be physically transported by the vehicle into positions where communication performance is sufficient [88]. Using path loss simulation software and optimisation techniques, the overall mission can be optimised given constraints on latencies and other mission parameters [89]. When optimising the mobility of network nodes, the communication network topology may also be autonomously and dynamically reconfigured, given the requirements of the different nodes at the time.

Another way of optimising the use of payload communication capacity versus other resources on-board vehicles, is by mission-oriented and autonomous on-board processing and analysis of the payload data. After basic pre-processing, the use of data compression is an obvious alternative if the end user requires relatively raw data for visualisation or processing in an operations centre [90]. Further reduction in communication requirements can be achieved by a higher level of autonomy where the on-board processing system analyses the data for detection, tracking, classification and recognition of objects of interest [91]. In complex network architectures, an in-network allocation of computational resources can be used in order to optimise system performance.

Navigation and communication systems often utilise similar technologies and can be combined. While primary navigation aids such as GNSS and hydro-acoustic positioning are often designed for navigation only, it is common that communication systems are used as secondary systems for navigation or aiding. Examples of this might be range (and possibly bearing and Doppler speed) measurements from radio receivers and communication of satellite fixes from surface assets to underwater assets.

In scientific missions, such as oceanography, the observation system might be be supported by oceanographic models that estimates and predicts the spatial and temporal evolution of the phenomena of interest. These can be linked to physical boundary conditions such as winds, waves and

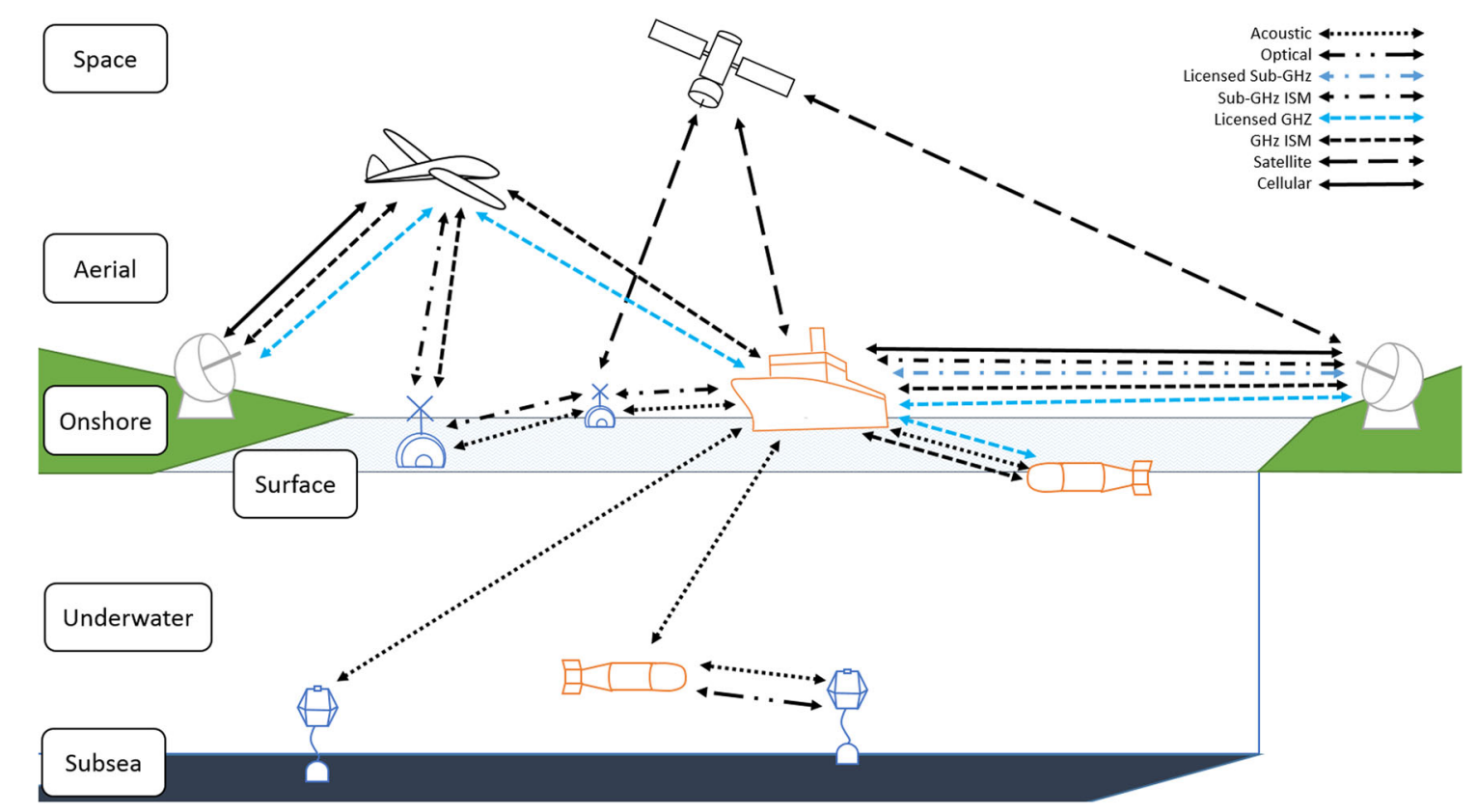

Fig. 6 Communication and Networking 
ocean currents. Usually, such models are computationally very complex, and may not be feasible to execute on-board small autonomous vehicles. Nevertheless, the information provided by such models may be of high value for the autonomous vehicle in order to optimise and plan its mission by taking into account the observations made by itself and other vehicles and sensors involved in the mission. In particular, such information would enable adaptive sampling behaviours that can greatly increase the efficiency of vehicle resource utilisation compared to, e.g. lawnmower patterns for search and mapping [92, 93].

Regarding communication systems, the standout difference between larger-scale and smaller-scale unmanned vehicles is that the former can afford high-throughput radio links. Communications power consumption can be negligible when compared with the overall running costs. Furthermore, the size of the vehicles may allow the use high-gain and directionally steerable antennas, and several different communication technologies. However, this is not the case for all autonomous unmanned vehicles. Next, this section discusses the appropriateness of different communication links, taking into account different vehicles and the medium where they may operate (i.e. air, space and underwater).

\subsection{Over the Air Communications}

For aerial and surface vehicles the most convenient way of exchanging information is by using electromagnetic waves. The single link communication performance depends on several parameters: the transmit power, link attenuation, transmit and receive antennas, available bandwidth, and the propagation environment. In addition, a communication system includes several links, that share a common medium, and must be coordinated in time, frequency and/or space.

Regarding used frequencies, many of the currently available technologies, especially those used for research, are based on license-free ISM frequency bands, slightly varying between countries. Some of the most commonly used frequencies for data transfer in unmanned vehicles are sub-GHz ISM ( $433 \mathrm{MHz}, 868 \mathrm{MHz}$ and $900 \mathrm{MHz})$ and $\mathrm{GHz}$ ISM $(2.4 \mathrm{GHz}$ and $5.8 \mathrm{GHz})$ [94]. In these spectra operate for example IEEE 802.11 or IEEE 802.15.4, as well as a great number of proprietary solutions.

The selection of appropriate communication technologies involves several trade-offs. These are often useful to consider in terms of a single link data-rate, and the induced interference experience by other links due to the transmission. With respect to the Shannon-Hartley theorem, the available bit rate of a single link is for all practical purposes equal to the product of channel's bandwidth and the logarithm of the received Signal to Noise Ratio (SNR) [95]. The induced interference depends on the transmit power and the attenuation towards the interfered receivers.
Considering the single link performance, the available bandwidth and allowed transmit power depend on the regulations, but the received power depends on the link attenuation and antennas utilised. The link attenuation increases with distance, and carrier frequency. As antennas are the remaining design consideration affecting the link attenuation, they are of primary interest to improve link performance and extend link coverage. Antenna directivity can improve link performance significantly, but is limited by the physical antenna size. Larger vehicles can be installed with a larger antenna or array, and can achieve better data-rates.

Link attenuation is, more precisely, the frequency response of the propagation environment, at the utilised communication frequencies. With a little simplification, we may derive intuition by considering if there exist major obstacles in or near the line of sight between the transmitter and receiver, blocking the propagation, and if there are objects that induce scattering of the radio waves, illuminated by the transmitting or receiving antennas, and that are near enough that the total propagation distance via them is not too large for the frequency. Then, respectively, we may experience shadowing or fading effects of the received signals, making the received signal strength depend on the exact positions of the transmitter and receiver. With mobility, they would then experience random-like signal strength fluctuation.

For higher frequencies, regulations allow much higher channel bandwidths and, therefore, higher data-rate, but the link attenuation is higher. Thus, it is more efficient to use the large bandwidth at high frequencies to short distance links, where it can be used to trade-off with received power. The created interference is also reduced due to the high attenuation. Similarly, the smaller attenuation at low frequencies helps to create links over longer distances, but will also create interference over larger distances. When several transmit-receive pairs are within (communication or interfering) range of each other, they need to share the channel either by time or frequency division. ${ }^{7}$ Time division can be applied over short-distances if the nodes can be synchronised, but otherwise, for instance in the longdistance narrowband communications, frequency division should be applied.

When possible, and the additional effort of obtaining a licensed band is acceptable, licensed transceivers with higher power allowances, wider bandwidths and the possibility to control interference, are preferred.

Depending on the implementation, transceivers may offer Point-to-Point, or Point-to-Multipoint connections,

\footnotetext{
${ }^{7}$ In the future, spatial processing based sharing may be possible, but this is still beyond current state of the art in all but a few special scenarios.
} 
supporting various number of clients, ad-hoc and mesh connectivity, or static configurations.

Several maritime scenarios often consider the use of WSN technologies in situations where low-power, lowbitrate and short distance (few hundred meters) communication is necessary. For example, in moored scenarios surface nodes have limited power, which favours WSN technologies over satellite communication, and low antenna elevation, being typically placed in an array of a few hundred meters from each other. However, since these nodes may collect various types of data, from simple water parameters to high resolution images, higher data-rate radios may be desirable, as well as longer ranges for larger scenarios. In order to improve both the data-rate and range, unmanned vehicles can be used. For instance, by using UAVs as elevated relay-node antennas, the performance of technologies such as IEEE 802.15.4 or other low-power radios $[52,53]$ can be improved, reducing the impact from waves or swaying when compared against surface to surface communications. The literature often also proposes the use of 802.16 networks, as a solution suitable for maritime environments [96-99]. For long-range, high-capacity, lowlatency maritime radio communication networks, the use of commercially available phased-array antenna systems operating in a $5 \mathrm{GHz}$ licensed band has been demonstrated as well $[54,83]$.

\subsection{Satellite Radio Communications}

If we consider satellite communications a special case of radio, it is characterised by long distance between transmitter and receiver, large coverage per transmission, and high latency.

The coverage makes satellites the primary choice to support large scale operations with connectivity, within the limitations of the latency. An operation relying on satellites must have a sufficiently high autonomy level to operate regardless of communication delays. The latency also puts a severe limitation on coordination of multiple transmissions, any systems where multiple nodes try to transmit small amounts of data are operating very inefficiently.

Regarding the communication on larger vessels, satellite Broadband Internet links (using Very-Small-Aperture Terminals, VSAT) and Cellular Network modems were used to exchange data between them. This allowed these vehicles to act as gateways to other devices, using their IEEE 802.11 links whenever available.

\subsection{Underwater Communications}

For communication between AUVs, and partially USVs, electromagnetic waves can be used in underwater environments. However, this is not a favourable method for data exchange in water-based environments since severe signal attenuation limits both range and data-throughput. For this reason, researchers and industries use more suitable technologies based on optical [63] and acoustic signals [100]. Underwater optical wireless communications can reach high bitrates, in the order of $\mathrm{Gb} / \mathrm{s}$, while having lowpower requirements, but are limited to short transmission ranges [101]. Even though AUVs and other vehicles may approach optical modems closely enough, the performance of these systems is influenced by temperature fluctuation, turbid water where suspended particles generate strong back scattering and severe absorption at optical frequency band.

Acoustics used for underwater communications is superior to electromagnetic and optical waves for larger distances [102], being able to transmit hundreds of bits for very long ranges (e.g. $1000 \mathrm{~km}$ ) [101]. However, acoustic waves for underwater communication are also influenced by oceanographic conditions, water surface and bottom boundaries, time variation and relative movement between the vehicles and surface waves that lead to undesirable effects such as time delay and Doppler spread. Underwater Acoustic (UWA) channels characterised as doubly-selective (time and frequency selective) channels are extremely challenging and the channel is also limited in bandwidth. The carrier frequency for UWA is much lower than RF communication and normally provides maximum data-rate of tens of $\mathrm{kb} / \mathrm{s}$ [103]. In order to overcome the challenges a variety of techniques have been proposed in underwater acoustic communication community [104] such as incoherent and coherent modulation, channel equalisation, Time Reversal Mirrors (TRM) and Passive-Phase Conjugation (PPC). Applying TRM and PPC intends to reduce the order of adaptive Decision Feedback Equaliser (DFE) [105] since those two techniques can help reduce delay spread of channel. However, PPC requires channel estimation which could consume high computational resources. Moreover TRM relies on a relatively stable channel state during two-way transmission, which can be difficult to satisfy due to long propagation delays. Orthogonal Frequency Division Multiplexing (OFDM) systems can perform simple equalisation in frequency domain and eliminate the need for complex time-domain equalisers. However, the performance by OFDM degrades with larger Doppler spread due to fast movement of the vehicles. Therefore, the channel needs to be estimated and intercarrier-interference equaliser needs to be applied. Within the extremely band-limited and frequency selective underwater channels, Coherent Multiple-Input, Multiple-Output OFDM (MIMO-OFDM) is considered as the most appropriate method for efficient and high data-rate communications.

In order to achieve a reliable and efficient communication, underwater sensor networks would be a good choice where sensor nodes collect information/data and then send it to a sink or command centre. CAPTURE was proposed 
in [106] provides an end-to-end communication architecture for an acoustic network of underwater vehicles, while a summary of the most recent advances of network design principles with emphasis on deployment, localisation, topology design and position-based routing for 3D ocean sensor network are provided in [107].

\subsection{Fitting Communication Links and Networking}

Upon the selection of the appropriate vehicles for each type of mission, preferred communication channels and networking technologies can also be chosen according to the scale of each vehicle and available resources. That can also be influenced by the desired data-transfer bitrate. Table 2 gives examples of available communication channels present in the literature for Maritime Environments.

Vehicles may have different tasks and available resources in a scientific sensing mission. In addition, they may also have different roles regarding communication and networking. Taking this into consideration, three main classes of communication links are envisaged, as depicted by Fig. 7. These classes are:

\section{Backbone links}

The first class considers $\mathrm{GHz}$ frequencies to support "backbone links", which are best suited for powerful nodes capable of covering large distances. These links are resource-demanding and may require specialised equipment (e.g. antennas and communication infrastructure in space and onshore), but guarantee high bitrates at large distances beyond hundreds of kilometres;

\section{Gateway links}

An intermediate class that uses GHz ISM frequencies for providing "gateway links" between different vehicles at reasonable distances (i.e. hundreds of metres), enabling them with high bitrates (i.e. hundreds of $\mathrm{Mb} / \mathrm{s}$ ) and without compromising energy efficiency. In this same class, but from an underwater perspective, optical links can also be considered as gateways, providing highbitrate (i.e. up to $\mathrm{Gb} / \mathrm{s}$ ) links, though at shorter distances;

\section{Local links}

The third class of communication links is envisaged for resource-constrained nodes, where communication follows a WSN or Internet of Things (IoT) fashion, establishing lower-bitrate connections with other nodes. For this purpose, sub-GHz and sub-GHz ISM frequencies can be used to enable energy efficient links while covering large distances, up to tens of kilometres. Regarding underwater communications, acoustic links have similar characteristics, providing lower data-rates than optical communication but longer ranges.

Although satellite communication is frequently used in a role of a backbone link, it does not meet available bitrate

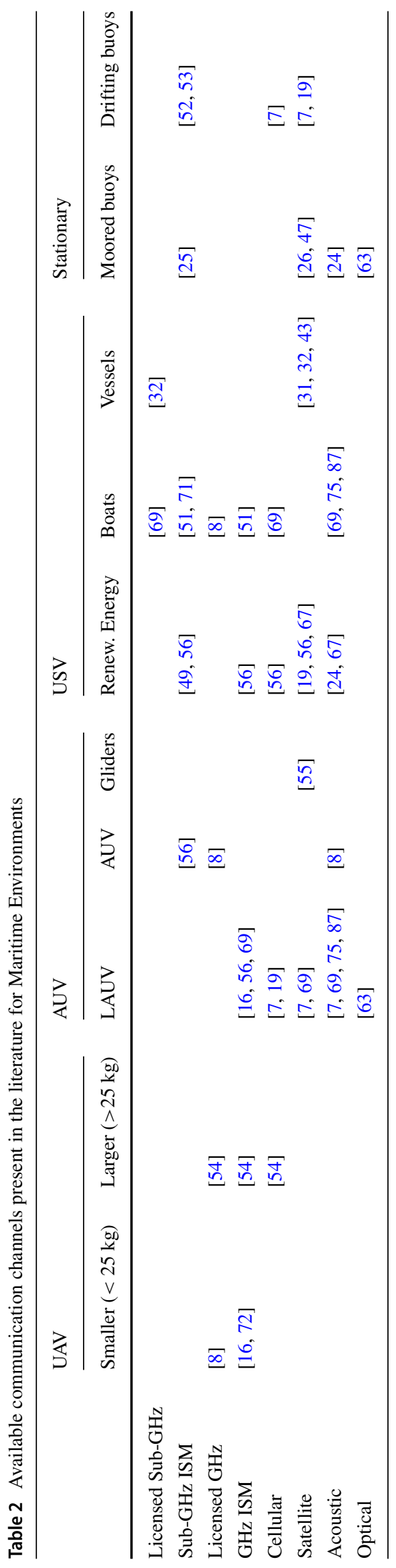




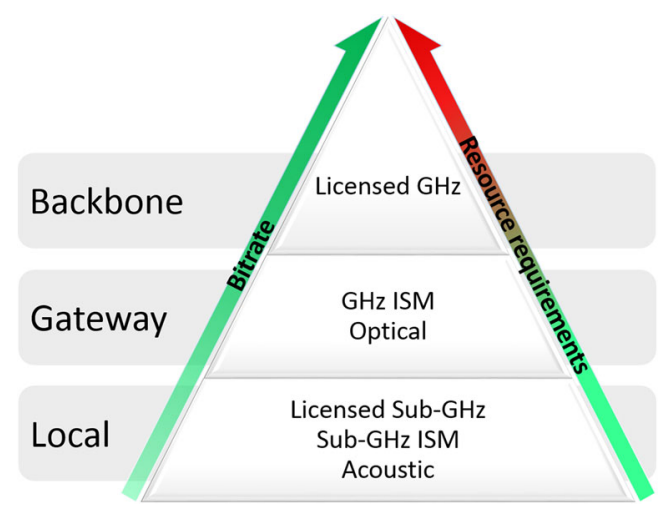

Fig. 7 Classes of communication links

requirements typical to backbone communication. For that reason satellite links are not considered in the above description, and on Fig. 7.

Focusing on vehicles' characteristics and diversity, UAVs may resort to different types of communication and fit into different classes. The main consideration is the size of the UAV and the desired coverage, which also influences the type of mission being planned.

For AUVs, underwater acoustic communications are suggested as a viable way of transmitting a small amount of data with a considerable range, suitable for Personal Area Network (PAN) links. Nonetheless, using GHz ISM links (i.e. Gateway links) provide the most efficient way of transmitting data when AUV resurface. Underwater optical communications can also be used to transmit data at higher bitrates, as Gateway links, but this requires shorter ranges, which are not suitable for Gliders, and their usage has not been fully exploited yet.

The deployment of arrays of moored buoys across large distances conveys them a characteristic that stands out from other nodes. Not only are they expected to operate over several years, but their isolation may also limit their interaction with other vehicles. This motivates the use of long-range links for establishing WSN/IoT connections (i.e. buoy to buoy), which are designed to not compromise resource-constrained devices' lifetime, but that the available bitrate to the order of $\mathrm{kb} / \mathrm{s}$. The preferred option for establishing Gateway links (i.e. high bitrate) is similar to AUVs, which should rely on GHz ISM communications above water and on optical modems when underwater, using other vehicles as relay nodes.

Large surface vessels have more resources and, similarly to large UAVs, are able to use more energy-demanding solutions such as $\mathrm{GHz}$ radios to achieve extended coverage and high bitrates. On the other hand, USVs based on energy harvesting are expected to operate for large periods of time and cover large distances, which renders $\mathrm{GHz}$ solutions, together with satellite-based communications, undesirable for relaying payload data. A more energy efficient solution is to exploit GHz ISM-based communication possibilities, exploiting Gateway links between planned or opportunistic visits of larger vehicles or UAVs, leaving expensive links for emergency situations only.

\subsection{Networks and Protocols}

Computer networks are present around the world, being composed of various protocols and communication technologies, creating an infrastructure that defines the Internet as we know it today. The ubiquitous presence of Internetcapable devices has, in the past few years, introduced the concept of the IoT, which aggregates any networkingcapable device under the assumption of common networking protocols, such as the Internet Protocol (IP).

Due to their importance, maritime environments should also be considered as part of the IoT, embracing a multitude of different unmanned vehicles and sensors, as well as of different communication technologies. In this context, the custom Inter-Module Communication (IMC) protocol as been proposed for interconnecting different systems [20]. This architecture allows the forwarding of messages between vehicles and provides access to the Internet through, e.g. a Hub [7]. Even though the IMC is not a standardised protocol, it is open-source and therefore can be used by anybody. All the acquired information can be seamlessly forwarded through devices using the LSTS Toolchain and directly visualised on their Neptus C4I (Command, Control, Communications, Computation and Intelligence) software, also open-source. The system's configuration is highly dependant on this toolchain, which requires a pre-configuration of the entire system. On the other hand, this system allows the use of heterogeneous technologies, such as satellite and acoustic links. Moreover, the architecture is decentralised, which provides robustness in autonomous systems where communication link availability may be intermittent. Another example of a software environment that enables the cooperation of autonomous vehicles is the Mission Oriented Operating Suite Interval Programming (MOOS-IvP) [117].

Aligned with the goals of aggregating different devices under a "common Internet", the Internet Engineering Task Force (IETF), ${ }^{8}$ responsible for standardising the Internet as we know it, hosted different Working Groups (WG) focused on handling this issue. Notable examples of such activities are IPv6 [118] and the IPv6 over Networks of Resource-constrained Nodes (6lo) WG, ${ }^{9}$ which are ideal for interconnecting heterogeneous networks such as the ones found in maritime scenarios.

\footnotetext{
${ }^{8}$ http://ietf.org

${ }^{9}$ https://datatracker.ietf.org/wg/6lo/
} 
The various research activities towards standardising the Internet of Things have allowed to establish a common architecture for different devices, with different capabilities, where IPv6 is seen as a convergence layer between several types of networks (e.g. Ethernet, IEEE 802.11, Bluetooth or $3 \mathrm{G} / 4 \mathrm{G} / 5 \mathrm{G}$, among others). Other WGs complete this common architecture by focusing on routing aspects, such as the Routing Over Low power and Lossy networks (ROLL) ${ }^{10}$ and in the definition of resource-oriented frameworks such as the Constrained RESTful Environments (CORE). ${ }^{11}$

Wireless Sensor Networks (WSNs) have been used in the past not only inland but also in different maritime environments [110, 111, 119, 120], and developments on WSNs have increased significantly as a subset of IoT [115]. In fact, several IoT communication technologies for Personal Area Networks (PANs) have been derived from WSNs, such as the IEEE 802.15.4 standard [121].

Due to the heterogeneity of applications and nodes in maritime environments, WSNs represent only a small group of networking possibilities to be considered. Their network architectures include the concept of sink nodes, or border routers, similarly to mesh networks, which typically act as gateways or as a backbone to the Internet. Alternatively, Mobile Ad-hoc Networks (MANETs) have a more distributed network architecture where routing protocols do not necessarily require nodes with particular roles [122]. MANETs have already been proposed for maritime environments [109, 123], but the characteristics, constraints and challenges of such environments require additional considerations such as Disruptive or Delay Tolerant Networking (DTN) and different routing protocols $[113,114,116]$.

Table 3 presents networking options used in the literature, regarding common communication technologies, and which may be applied to various types of autonomous vehicles.

When considering autonomous system challenges and off-the-shelf equipment, specific solutions for creating a network of heterogeneous vehicles in maritime environments have been developed in the past [20, 108]. As an example, a solution that provides interoperability between different communication technologies and motivates the employment of features, such as DTN, is presented in [108]. Even though this solution relies on custom protocols, it opens the possibility of creating a standardised link to the future Internet, where IPv6 and 6LoWPAN can be used to interconnect devices with different capabilities and solve issues such as automatic address attribution [124].

The high number of available maritime nodes already deployed around the world should also promote cooperation

\footnotetext{
${ }^{10} \mathrm{https}: / /$ tools.ietf.org/wg/roll

${ }^{11} \mathrm{https} / / /$ datatracker.ietf.org/wg/core/
}

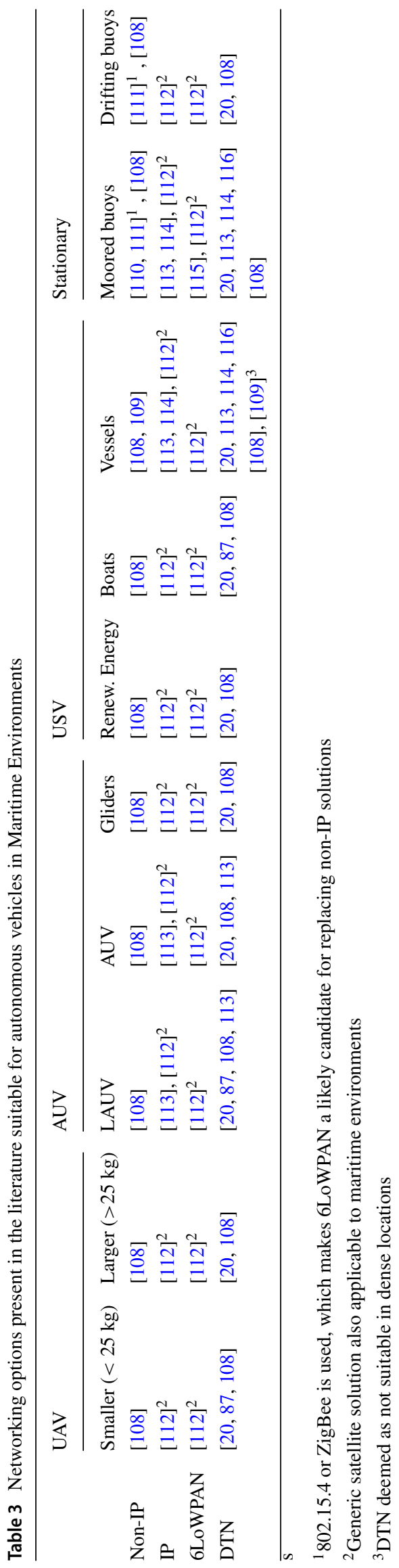


between them, creating a network for different manned or unmanned operations. However, this is still not a reality, partly due to not applying standardised protocols. From the mission planning point-of-view, the automatic configuration of network-capable nodes is a desirable feature, where address auto-configuration features from IPv6, as well as low-overhead routing protocols, could be exploited. This would enable visiting vehicles, or new nodes, to seamlessly integrate existing networks which could increase vehicles and nodes capabilities and improve the coverage. In addition to allowing the deployment of new nodes in a "plug and net" fashion, such an automatic configuration of the network would also be important to adapt the network topology (i.e. routing paths), as the network topology will likely change with time - Line-of-Sight (LOS) can be obstructed by vessels, or sea state - maintaining a dynamic mesh or hierarchical network.

Similarly to the Internet infrastructure and architecture, powerful and resourceful nodes such as ships could have an important role as possible coordinator points, or as aggregation units. For example, large-scale vehicles could act as routers or gateways, capable of providing a backbone link between other systems, or even directly to the Internet. A fully networked system of unmanned vehicles using standardised protocols and operations would allow control centres to navigate vehicles, and users to analyse environmental data across the world, connected to the Internet.

The use of IPv6 in maritime networks will allow interoperability with the Internet of Things, connecting them with others, such as satellite networks [112], and will provide a large number of mature and standardised features that build on IP (e.g. security and reliability). Desirable features include not only the routing and multihop routing between different nodes and technologies, but also the possibility to handle distinct data flows, with specific requirements (e.g. control data vs. payload data), in different ways [125]. For instance, traffic engineering in maritime environments allows mapping data-flows to different technologies and routes, taking available network resources, cost and data characteristics (e.g. priority) into account.

Regarding future network management and control, Software-Defined Networking (SDN) can be seen as a means for simplification and evolvability of existing

Table 4 Summary of Technologies suggested for Maritime Environments

\begin{tabular}{|c|c|c|c|c|c|c|c|c|c|c|}
\hline & \multicolumn{2}{|l|}{ UAV } & \multicolumn{3}{|l|}{ AUV } & \multicolumn{3}{|l|}{ USV } & \multicolumn{2}{|c|}{ Stationary } \\
\hline & $\begin{array}{l}\text { Smaller } \\
(<25 \mathrm{~kg})\end{array}$ & $\begin{array}{l}\text { Larger } \\
(>25 \mathrm{~kg})\end{array}$ & LAUV & AUV & Gliders & $\begin{array}{l}\text { Renew. } \\
\text { Energy }\end{array}$ & Boats & Vessels & $\begin{array}{l}\text { Moored } \\
\text { buoys }\end{array}$ & $\begin{array}{l}\text { Drifting } \\
\text { buoys }\end{array}$ \\
\hline Small-scale $(0-10 \mathrm{~km})$ & $\checkmark$ & & $\checkmark$ & $\checkmark$ & & & $\checkmark$ & & $\checkmark$ & $\checkmark$ \\
\hline Medium-scale (10-100 km) & & $\checkmark$ & & $\checkmark$ & $\checkmark$ & $\checkmark$ & $\checkmark$ & & $\checkmark$ & $\checkmark$ \\
\hline Large-scale (100-1000 km) & & $\checkmark$ & & & $\checkmark$ & $\checkmark$ & & $\checkmark$ & $\checkmark$ & $\checkmark$ \\
\hline Global-scale (>1000 km) & & $\checkmark$ & & & $\checkmark$ & $\checkmark$ & & $\checkmark$ & $\checkmark$ & $\checkmark$ \\
\hline \multicolumn{11}{|c|}{ Available Communication Channels } \\
\hline Licensed Sub-GHz & & & & & & & & & $\checkmark^{1}$ & \\
\hline Sub-GHz ISM & & & & & & & & & $\checkmark^{1}$ & \\
\hline Licensed GHz & & $\checkmark$ & & & & & & $\checkmark$ & & \\
\hline GHz ISM & $\checkmark$ & $\checkmark$ & $\checkmark$ & $\checkmark$ & $\checkmark$ & $\checkmark$ & $\checkmark$ & & $\checkmark$ & $\checkmark$ \\
\hline Cellular & & $\checkmark^{2,3}$ & $\checkmark^{2}$ & & & $\checkmark^{2}$ & $\checkmark^{2}$ & & $\checkmark^{2}$ & \\
\hline Satellite & & $\checkmark^{3}$ & $\checkmark^{3}$ & $\checkmark^{1}$ & $\checkmark^{1}$ & $\checkmark^{1}$ & $\checkmark^{3}$ & $\checkmark^{1}$ & $\checkmark^{1}$ & $\checkmark^{1}$ \\
\hline Acoustic & & & $\checkmark^{1}$ & $\checkmark^{1}$ & $\checkmark^{1}$ & & & & $\checkmark^{1}$ & $\checkmark^{1}$ \\
\hline Optical & & & $\checkmark$ & $\checkmark$ & & & & & $\checkmark$ & $\checkmark$ \\
\hline \multicolumn{11}{|l|}{ Networking Options } \\
\hline IPv6 & $\checkmark$ & $\checkmark$ & $\checkmark$ & $\checkmark$ & $\checkmark$ & $\checkmark$ & $\checkmark$ & $\checkmark$ & $\checkmark$ & $\checkmark$ \\
\hline 6LoWPAN & & & $\checkmark^{1}$ & $\checkmark^{1}$ & $\checkmark^{1}$ & & & & $\checkmark^{1}$ & $\checkmark^{1}$ \\
\hline DTN/SDN & $\checkmark$ & $\checkmark$ & $\checkmark$ & $\checkmark$ & $\checkmark$ & $\checkmark$ & $\checkmark$ & $\checkmark$ & $\checkmark$ & $\checkmark$ \\
\hline
\end{tabular}

\footnotetext{
${ }^{1}$ Resource-constrained solutions that have limited bitrates and require specific protocols (e.g. IoT/WSN protocols), but that should still be considered to complement the unavailability of better "gateway links" (e.g. GHz-ISM or Optical)

${ }^{2}$ Only if operated in an areas of sufficient network coverage

${ }^{3}$ Only as a backup-link
} 
networking solutions [126]. The SDN approach enables a separation of the control plane from the data plane into a central controller, which also opens new possibilities for demanding scenarios such as maritime environments and WSNs [127]. In fact, by optimising the number of signalling messages from fully distributed protocols, as well as by migrating computationally complex algorithms to the control plane, improvements on scalability and energy savings may be achieved. Moreover, the SDN paradigm allows a fine-grained control of data-flows, enabling sophisticated traffic management options such as prioritisation or multiple backup paths.

\section{Trends for Maritime Communication and Networking of Autonomous Vehicles}

Table 4 summarises the available options regarding the use of unmanned vehicles for different remote-sensing operations, taking into account the scale or range of the area to be surveyed. The characteristics of each vehicle influence not only their use in specific conditions, but also the type of communication technologies that can be accommodated. For example, underwater radio communication is deemed as not likely to be used in any of the defined scales of operation.

The chosen communication technologies represent the primary data-links per vehicle, as previously discussed, exploiting interoperability and interactions between different devices for conserving available resources and relaying data between more-powerful vehicles. In particular, significant resource-constraints that result from vehicles' specifics (e.g. available energy, typical lifetime) are illustrated by the networking and communication options with a superscript marker. These correspond to resource-constrained solutions that have limited bitrates and require specific protocols (e.g. IoT/WSN protocols), but that should still be considered to complement the unavailability of better "gateway links" (e.g. GHz-ISM or Optical).

The conceptualised interconnection mechanisms between the vehicles are supported by IPv6, where its compressed version (6LoWPAN) is used for resourceconstrained links. Cyber-security, hijacking, piracy and related issues are of considerable concern in unmanned vehicles operations. The IP-based approach allows the use of virtually any existing networking solution, enabling features such as security, which have been disregarded by previous works. The use of networking functionalities such as delay-tolerant and software-defined networking are also seen as desirable across all types of vehicles, but further work needs to be conducted in this direction.

\section{Conclusions}

The importance of autonomous marine systems is undeniable and the opportunity for coordinated and interconnected operations is clear.

Operations may take place in remote locations with long distance to the operations centre, so that dependence on third-party infrastructures such as satellite communication or terrestrial communication systems must be expected. The cost, reliability, performance and availability of such systems are important issues.

Moreover, there is a wide variety of data with different communication requirements with respect to data-rates, latency and importance. These are for instance, Command and control data (telemetry), sensor data for situation awareness, payload sensor data, collision avoidance transponder broadcasts and status information. Therefore, communication requirements strongly depend on the system autonomy level and user needs.

This work reviews the major advancements on maritime technology applied in several different scenarios, from transportation to scientific research. Moreover, it highlights how available technologies can be composed in order to efficiently and effectively operate in maritime environments.

Existing and prototype unmanned vehicles, sensors and communication technologies are characterised, describing their requirements and capabilities. Additionally, the tradeoff between fully autonomous operations versus remotely operated vehicles is highlighted, taking into account the availability and performance of different communication options. The discussed opportunities are aligned with current trends in networking and communication technologies.

Acknowledgements This work was partially funded by the European Union's Horizon 2020 research and innovation programme under the Marie Sklodowska-Curie Grant Agreement No. 699924.

The Research Council of Norway is acknowledged as the main sponsor of NTNU AMOS (Centre for Autonomous Marine Operations and Systems), grant number 223254, and the project Hybrid Operations in Maritime Environments (HOME) funded by the MAROFF programme, grant number 269480.

The work has been supported by the light house project CAMOS of the Faculty of Information Technology and Electrical Engineering, NTNU.

Open Access This article is distributed under the terms of the Creative Commons Attribution 4.0 International License (http:// creativecommons.org/licenses/by/4.0/), which permits unrestricted use, distribution, and reproduction in any medium, provided you give appropriate credit to the original author(s) and the source, provide a link to the Creative Commons license, and indicate if changes were made.

Publisher's Note Springer Nature remains neutral with regard to jurisdictional claims in published maps and institutional affiliations. 


\section{References}

1. Campbell, N.: Biology: Concepts \& Connections. Pearson/Benjamin Cummings (2006), https://books.google.com.ng/ books?id=OhdFAQAAIAAJ

2. European Union: The blue economy of the european union. http://ec.europa.eu/maritimeaffairs/documentation/publications/ documents/poster-blue-growth-2015_en.pdf (2015), [Online; Accessed 13 Jul 2016]

3. Woodget, A.S., Carbonneau, P.E., Visser, F., Maddock, I.P.: Quantifying submerged fluvial topography using hyperspatial resolution uas imagery and structure from motion photogrammetry. Earth Surface Process Landforms 40(1), 47-64 (2015). https://doi.org/10.1002/esp.3613

4. Sivertsen, A., Solb $\varnothing$, S., Storvold, R., Tøllefsen, A., Johansen, K.S.: Automatic mapping of sea ice using unmanned aircrafts. In: ReCAMP Flagship Workshop Book of Abstracts. p. 30. ReCAMP Flagship Workshop, ReCAMP Flagship Workshop (2016), n/a

5. Lucieer, A., Turner, D., King, D.H., Robinson, S.A.: Using an unmanned aerial vehicle (uav) to capture micro-topography of antarctic moss beds. Int. J. Applied Earth Observ. Geoinform. Part A 27, 53-62 (2014). http://www.sciencedirect.com/science/ article/pii/S0303243413000603 special Issue on Polar Remote Sensing (2013)

6. Solbø, S., Storvold, R., Sivertsen, A., Petrich, C., Sand, B.: Imaging sea ice structure by small remotely piloted aircraft. In: ReCAMP Flagship Workshop Book of Abstracts. p. 32. ReCAMP Flagship Workshop, ReCAMP Flagship Workshop (2016), n/a

7. Faria, M., Pinto, J., Py, F., Fortuna, J., Dias, H., Martins, R., Leira, F., Johansen, T.A., Sousa, J., Rajan, K.: Coordinating uavs and auvs for oceanographic field experiments: Challenges and lessons learned experiments in uav and auv control for coastal oceanography. In: IEEE Int. Conf. Robotics and Automation. Hong Kong (2014)

8. Ludvigsen, M., Dias, P.S., Ferreira, S., Fossum, T.O., Hovstein, V., Johansen, T.A., Krogstad, T.R., Midtgaard, Ø., Norgren, P., J.S., Sture, Ø., Vågsholm, E., Zolich, A.: Autonomous network of heterogeneous vehicles for marine research and management. In: IEEE Oceans 2016 - Monterey (2016)

9. Py, F., Pinto, J., Silva, M.A., Johansen, T.A., Sousa, J., K., R.: Europtus: A mixed-initiative controller for multi-vehicle oceanographic field experiments. In: Int. Symp. Experimental Robotics (2016)

10. Roemmich, D., Boehme, L., Claustre, H., Freeland, H., Fukasawa, M., Goni, G., Gould, W.J., Gruber, N., Hood, M., Kent, E., Lumpkin, R., Smith, S., Testor, P.: Integrating the ocean observing system: Mobile platforms. In: Proceedings of OceanObs'09: Sustained Ocean Observations and Information for Society. European Space Agency (2010) https://doi.org/10.5270/OceanObs09. pp.33

11. Greene, C.H., Meyer-Gutbrod, E.L., McGarry, L.P. Jr., L.C.H., McClatchie, S., Packer, A., Jung, J.B., Acker, T., Dorn, H., Pelkie, C.: A wave glider approach to fisheries acoustics: Transforming how we monitor the nation's commercial fisheries in the 21 st century. Oceanography 27 . https://doi.org/10.5670/ oceanog.2014.82 (2014)

12. Perry, M.J., Rudnick, D.L.: Observing the ocean with autonomous and lagrangian platforms and sensors: The role of alps in sustained ocean observing systems. Oceanography 16(4), 31-36 (2003). n/a

13. Dawson, C.: Arctic shipping volume rises as ice melts. http:// www.wsj.com/articles/arctic-cargo-shipping-volume-is-rising-asice-melts-1414612143 (2014), [Online; Accessed 15 May 2016]
14. Trishchenko, A.P., Garand, L.: Continuous coverage of the arctic: Two-satellite highly elliptical orbit (heo) system is better than two dozen of leo polar orbiters, http://www.goes-r.gov/downloads/ 2012-Science-Week/posters/tues/13_Trishchenko.pdf

15. Imagenex Technologies: Overview of the imagenex deltat sonar real time operation in an autonomous underwater vehicle (auv) application (2006)

16. Johansen, T.A., Zolich, A., Hansen, T., Sørensen, A.J.: Unmanned aerial vehicle as communication relay for autonomous underwater vehicle - field tests. In: IEEE Globecom Workshop - Wireless Networking and Control for Unmanned Autonomous Vehicles. Austin (2014)

17. Zolich, A., Johansen, T.A., Cisek, K., Klausen, K.: Unmanned aerial system architecture for maritime missions. Design and hardware description. In: 2015 Workshop on Research, Education and Development of Unmanned Aerial Systems (RED-UAS). pp. 342-350 (2015)

18. Maritime, K.: Reshaping underwater operations - live footage of groundbreaking robotic subsea 'snake' released, https://www.km. kongsberg.com/ks/web/nokbg0238.nsf/AllWeb/81FA0DE33AE2 C0FAC12580CA0037AD9C?OpenDocument

19. Sousa, L.L., López-Castejón, F., Gilabert, J., Relvas, P., Couto, A., Queiroz, N., Caldas, R., Dias, P.S., Dias, H., Faria, M., Ferreira, F., Ferreira, A.S., Fortuna, J., Gomes, R.J., Loureiro, B., Martins, R., Madureira, L., Neiva, J., Oliveira, M., Pereira, J., Pinto, J., Py, F., Queirós, H., Silva, D., Sujit, P., Zolich, A., Johansen, T.A., Sousa, J., Rajan, K.: Integrated monitoring of mola mola behaviour in space and time. PLoS ONE (2016)

20. Pinto, J., Dias, P.S., Martins, R., Fortuna, J., Marques, E., Sousa, J.: The lsts toolchain for networked vehicle systems. In: OCEANS - Bergen, 2013 MTS/IEEE. pp. 1-9 (June 2013)

21. Ocean, G.: Ocean tracking network global metadata and data atlas. http://members.oceantrack.org/data/discovery/GLOBAL. htm (2016), [Online; Accessed 13 July 2016]

22. Argo: How argo floats work, http://www.argo.ucsd.edu/How Argo_floats.html

23. M., D.: Methods for the deployment and maintenance of an acoustic tag tracking array: An example from california's channel islands. Mar. Technol. Soc. J. 39, 74-80 (2005)

24. Beauchamp, N.: Return of the intrepid wave glider. http:// oceantrackingnetwork.org/return-of-the-intrepid-wave-glider/ (2014), [Online; Accessed 13 July 2016]

25. Zolich, A., Skøien, K.R., Alfredsen, J.A., Johansen, T.A.: A communication bridge between underwater sensors and unmanned vehicles using a surface wireless sensor network design and validation. In: IEEE Oceans Shanghai (2016)

26. Berge, J., Geoffroy, M., Johnsen, G., Cottier, F., Bluhm, B., Vogedes, D.: Ice-tethered observational platforms in the arctic ocean pack ice. IFAC-PapersOnLine 49(23), 494499 (2016). http://www.sciencedirect.com/science/article/pii/ S2405896316320742

27. Fossen, T.I.: Project 5 - autonomous aerial systems for marine monitoring and data collection. http://www.ntnu.edu/ amos/project-5 (2016), [Online; Accessed 13 July 2016]

28. Ltd., H.A.V.: Access to the arctic: Time to try something different - press release (2013), https://www.hybridairvehicles. com/downloads/Airlander-220.pdf

29. DNV-GL: The revolt - a new inspirational ship concept (2015) https://www.dnvgl.com/technology-innovation/revolt/index.html

30. Rolls-Royce Marine: Rolls-royce drone ships challenge $\$ 375$ billion industry: Freight (2014) http://www.bloomberg.com/news/ articles/2014-02-25/rolls-royce-drone-ships-challenge-375-billionindustry-freight 
31. Rolls-Royce: Rolls-royce unveils a vision of the future of remote and autonomous shipping. http://www.rolls-royce.com/ media/press-releases/yr-2016/pr-12-04-2016-rr-unveils-a-visionof-future-of-remote-and-autonomus-shipping.aspx (2016), [Online; Accessed 13 July 2016]

32. Rødseth, Ø.J., Kvamstad, B., Porathe, T., Burmeister, H.C.: Communication architecture for an unmanned merchant ship. In: IEEE Oceans. Bergen (2013)

33. Rolls-Royce Marine: Rolls-royce unveils a vision of the future of remote and autonomous shipping (2016) http://www.rolls-royce. com/media/press-releases/yr-2016/pr-12-04-2016-rr-unveils-avision-of-future-of-remote-and-autonomus-shipping.aspx

34. World, Y.B.: Finferries' 65 metre double ended ferry, the stella will be used to test how crewless ships function in a real environment (2016) http://www.ybw.com/pictures/rolls-royce-crew less-smart-boats-18760/attachment/26248512775_8e66afa2b0_o

35. Elkins, L., Sellers, D., Monach, W.R.: The autonomous maritime navigation ( $\mathrm{amn}$ ) project: Field tests, autonomous and cooperative behaviors, data fusion, sensors and vehicles. J. Field Robot. 27, 790-818 (2010)

36. Wolf, M.T., Assad, C., Kuwata, Y., Howard, A., Aghazarian, H., Zhu, D., Lu, T., Trebl-Ollennu, A., Huntsberger, T.: 360-degree visual detection and target tracking on an autonomous surface vehicle. J. Field Robot. 27, 819-830 (2010)

37. Huntsberger, T., Aghazarian, H., Howard, A., Trotz, D.C.: Stereo vision-based navigation for autonomous surface vessels. J. Field Robot. 28, 3-18 (2011)

38. Kuwata, Y., Wolf, M.T., Zarzhitsky, D., Huntsberger, T.L.: Safe maritime autonomous navigation with COLREGS, using velocity obstacles. IEEE J. Oceanic Eng. 39, 110-119 (2014)

39. COLREGs - convention on the international regulations for preventing collisions at sea, international maritime organization (IMO) (1972)

40. Stensvold, T.: Rolls-royce bygger fjernstyringssenter i Ålesund (2017), https://www.tu.no/artikler/rolls-royce-bygger-fjernstyrings senter-i-alesund/377772

41. Turku, U.: Aawa - advanced autonomous waterborne applications initiative. https://www.utu.fi/en/units/law/research/researchprojects/Pages/aawa.aspx (2015), [Online; Accessed 13 July 2016]

42. Hannu, K.: Rolls-royce and vtt unveil a vision of ship intelligence with futuristic ox bridge concept. http://www.vttresearch.com/ media/news/rolls-royce-and-vtt-unveil-a-vision-of-ship-intelligencewith-futuristic-ox-bridge-concept (2014), [Online; Accessed 13 July 2016]

43. MUNIN Consortium: Research in maritime autonomous systems project results and technology potentials. http://www.unmannedship.org/munin/wp-content/uploads/2016/02/MUNIN-final-broc hure.pdf (2016), [Online; Accessed 13 July 2016]

44. Johansen, T.A., Perez, T.: Unmanned aerial surveillance system for hazard collision avoidance in autonomous shipping. In: International Conference on Unmanned Aircraft Systems, Washington DC (2016)

45. Kongsberg: Collaboration on swimming robots for subsea maintenance. https://www.km.kongsberg.com/ks/web/nokbg0238.nsf/ AllWeb/2800489E780D5865C1257F99002DCDA6?OpenDocu ment(2016), [Online; Accessed 13 July 2016]

46. Guerra, A.G., Francisco, F., Villate, J., Agelet, F.A., Bertolami, O., Rajan, K.: On small satellites for oceanography: A survey. Acta Astronautica 127, 404-423 (2016). http://www. sciencedirect.com/science/article/pii/S0094576515303441

47. Osse, T.J., Meinig, C., Stalin, S., Milburn, H.: The prawler, a vertical profiler powered by wave energy. In: OCEANS 2015 MTS/IEEE Washington. pp. 1-8 (2015)
48. Domeier, M.L.: Methods for the deployment and maintenance of an acoustic tag tracking array: An example from california's channel islands. Marine Technol Soc J 39(1), 74-80 (2005). https://doi.org/10.4031/002533205787521668

49. Dunbabin, M., Grinham, A.: Experimental evaluation of an autonomous surface vehicle for water quality and greenhouse gas emission monitoring. In: 2010 IEEE International Conference on Robotics and Automation. pp. 5268-5274 (2010)

50. Caccia, M., Bibuli, M., Bono, R., Bruzzone, G.: Basic navigation, guidance and control of an unmanned surface vehicle. Auton. Robot. 25, 349-365 (2008)

51. Kimball, P., Bailey, J., Das, S., Geyer, R., Harrison, T., Kunz, C., Manganini, K., Mankoff, K., Samuelson, K., Sayre-McCord, T., Straneo, F., Traykovski, P., Singh, H.: The whoi jetyak: An autonomous surface vehicle for oceanographic research in shallow or dangerous waters. In: 2014 IEEE/OES Autonomous Underwater Vehicles (AUV). pp. 1-7 (2014)

52. Barbatei, R., Skavhaug, A., Johansen, T.A.: Acquisition and relaying of data from a floating wireless sensor node using an unmanned aerial vehicle. In: 2015 International Conference on Unmanned Aircraft Systems (ICUAS), pp. 677-686 (2015)

53. Palmer, J., Yuen, N., Ore, J.P., Detweiler, C., Basha, E.: On airto-water radio communication between uavs and water sensor networks. In: 2015 IEEE International Conference on Robotics and Automation (ICRA), pp. 5311-5317 (2015)

54. Hovstein, V.E., Sægrov, A., Johansen, T.A.: Experiences with coastal and maritime UAS BLOS operation with phased-array antenna digital payload data link. In: Int. Conf. Unmanned Aerial Systems (ICUAS). Orlando (2014)

55. Sherman, J., Davis, R.E., Owens, W.B., Valdes, J.: The autonomous underwater glider "spray". IEEE J. Ocean. Eng. 26(4), 437-446 (2001)

56. O'Reilly, T.C., Kieft, B., Chaffey, M.: Communications relay and autonomous tracking applications for wave glider. In: OCEANS 2015 - Genova (2015)

57. Gizmag: Darpa readies unmanned actuv sub hunter for sea trials (2016), http://www.gizmag.com/darpa-actuv-unmanned-sub-hun ter/41842/

58. Yuh, J.: Design and control of autonomous underwater robots: A survey. Auton. Robot. 8, 7-24 (2000)

59. Zheng, H., Negenborn, R.R., Lodewijks, G.: Survey of approaches for improving the intelligence of marine surface vehicles. In: 16th International IEEE Conference on Intelligent Transportation Systems (ITSC 2013). pp. 1217-1223 (2013)

60. Fernandes, P.G., Stevenson, P., Brierley, A.S., Armstrong, F., Simmonds, E.: Autonomous underwater vehicles: future platforms for fisheries acoustics. ICES J Mar Sci 60(3), 684 (2003). https://doi.org/10.1016/S1054-3139(03)00038-9

61. Cui, J.H., Kong, J., Gerla, M., Zhou, S.: The challenges of building mobile underwater wireless networks for aquatic applications. IEEE Netw 20(3), 12-18 (2006)

62. Rajan, K., Py, F.: T-rex: Partitioned inference for auv mission control. In: Further Advances in Unmanned Marine Vehicles. pp. 171-199. Control, Robotics \& Sensors, Institution of Engineering and Technology (2012), http://digital-library.theiet. org/content/books/10.1049/pbce077e_ch9

63. Vasilescu, I., Kotay, K., Rus, D., Dunbabin, M., Corke, P.: Data collection, storage, and retrieval with an underwater sensor network. In: Proceedings of the International Conference on Embedded Networked Sensor Systems (ACM) SenSys 2005 (2005)

64. Desa, E., Maurya, P.K., Pereira, A., Pascoal, A.M., Prabhudesai, R.G., Mascarenhas, A., Desa, E., Madhan, R., Matondkar, S.G.P., Navelkar, G., Prabhudesai, S., Afzulpurkar, S.: A small 
autonomous surface vehicle for ocean color remote sensing. IEEE J. Ocean. Eng. 32(2), 353-364 (2007)

65. Manley, J.E.: Unmanned surface vehicles, 15 years of development. In: IEEE/MTS Oceans. Quebec City (2008)

66. Offshore-technology.com: Using autonomous vehicles to track ice in iceberg alley (2015), http://www.offshore-technology.com/

67. Hine, R., Willcox, S., Hine, G., Richardson, T.: The wave glider: A wave-powered autonomous marine vehicle. In: OCEANS 2009, pp. 1-6 (2009)

68. Global, A.: Asv global world leading marine autonomy (2017), http://asvglobal.com/

69. Norgren, P., Ludvigsen, M., Ingebretsen, T., Hovstein, V.E.: Tracking and remote monitoring of an autonomous underwater vehicle using an unmanned surface vehicle in the trondheim fjord. In: OCEANS 2015 - MTS/IEEE Washington. pp. 1-6 (2015)

70. Djapic, V., Na: Collaborative autonomous vehicle use in mine countermeasures. Sea Technology Magazine (2010) http://www. sea-technology.com/features/2010/1110/autonomous_vehicle.php

71. Zhang, J., Xiong, J., Zhang, G., Gu, F., He, Y.: Flooding disaster oriented usv uav system development demonstration. In: OCEANS 2016 - Shanghai, pp. 1-4 (2016)

72. Mendonça, R., Marques, M.M., Marques, F., Lourenço, A., Pinto, E., Santana, P., Coito, F., Lobo, V., Barata, J.: A cooperative multi-robot team for the surveillance of shipwreck survivors at sea. In: OCEANS 2016 MTS/IEEE Monterey, pp. $1-6(2016)$

73. Fan, Y., Ma, J., Wang, G., Li, T.: Design of a heterogeneous marsupial robotic system composed of an usv and an uav. In: 2016 Eighth International Conference on Advanced Computational Intelligence (ICACI), pp. 395-399 (2016)

74. Warwick, G.: Hybrid vtol uavs - back to the future. http://avia tionweek.com/blog/hybrid-vtol-uavs-back-future (2014), [Online; Accessed 13 July 2016]

75. Sarda, E.I., Dhanak, M.R.: A usv-based automated launch and recovery system for auvs. IEEE J. Ocean. Eng. 42(1), 37-55 (2017)

76. de Sousa, J.B., McGuillivary, P., Vicente, J., Bento, M.N., Morgado, J.A.P., Matos, M.M., Bencatel, R.A.G., de Oliveira, P.M.: Handbook of Unmanned Aerial Vehicles, chap. Unmanned Aircraft Systems for Maritime Operations, pp. 2787-2811. Springer, Netherlands (2015)

77. Usbeck, K., Gillen, M., Loyall, J., Gronosky, A., Sterling, J., Kohler, R., Newkirk, R., Canestrare, D.: Data ferrying to the tactical edge: A field experiment in exchanging mission plans and intelligence in austere environments. In: 2014 IEEE Military Communications Conference, pp. 1311-1317 (2014)

78. Chamberlain, L., Scherer, S.: Robocopters to the rescue. IEEE Spectr. 50(10), 28-33 (2013)

79. Parasuraman, R., Sheridan, T.B., Wickens, C.D.: A model for types and levels of human interaction with automation. IEEE Trans. Syst. Man, Cybern.- Part A: Syst. Humans 30(3), 286-297 (2000)

80. Register, L.: Shipright design and constructin, additional design procedures: Lr code for unmanned marine systems. Tech. rep., Lloyd's Register Group Limited (2 2017), http://www.lr.org/en/ services/unmanned-code.aspx

81. Council, N.R.: Review of ONR's Uninhabited Combat Air Vehicles Program. National Academies Press, Washington DC (2000)

82. International, S.: U.S. department of transportation's new policy on automated vehicles adopts sae international's levels of automation for defining driving automation in on-road motor vehicles (1 2014) https://www.sae.org/news/3544/
83. Zolich, A., Johansen, T.A., Sægrov, A., Vågsholm, E., Hovstein, V.: Coordinated maritime missions of unmanned vehicles network architecture and performance analysis. In: IEEE ICC, Mobile and Wireless Networking. Paris (2017)

84. Grancharova, A., Grøtli, E.I., Ho, D.T., Johansen, T.A.: UAVs, trajectory planning by distributed MPC under radio communication path loss constraints. J. Intell. Robot. Syst. 79, 115-134 (2015)

85. Grancharova, A., Grøtli, E.I., Johansen, T.A.: Rotary-wing uavs path planning by distributed linear MPC with reconfigurable communication network topologies. In: IFAC Workshop on Distributed Estimation and Control in Networked Systems. Koblenz (2013)

86. Ho, D.T., Grøtli, E.I., Sujit, P.B., Johansen, T.A., Sousa, J.: Optimization of wireless sensor network and UAV, data acquisition. J. Intell. Robot. Syst. 78, 159-179 (2015)

87. McGillivary, P., de Sousa, J.B., Martins, R., Rajan, K., Leroy, F.: Integrating autonomous underwater vessels, surface vessels and aircraft as persistent surveillance components of ocean observing studies. In: 2012 IEEE/OES Autonomous Underwater Vehicles (AUV), pp. 1-5 (2012)

88. Frew, E.W., Brown, T.X., Dixon, C., Henkel, D.: Establishment and maintenance of a delay tolerant network through decentralized mobility control. In: Proc. of the IEEE International Conference on Networking, Sensing and Control, pp. 584-589 (2006)

89. Grøtli, E.I., Johansen, T.A.: Motion- and communicationplanning of unmanned aerial vehicles in delay tolerant network using mixed-integer. Linear Program. Model. Identif. Control 37(2), 77-97 (2016)

90. Razif, M.A.M., Mokji, M., Zabidi, M.M.A.: Low complexity maritime surveillance video using background subtraction on h.264. In: 2015 International Symposium on Technology Management and Emerging Technologies (ISTMET), pp. 364-368 (2015)

91. Leira, F., Johansen, T.A., Fossen, T.I.: Automatic detection, classification and tracking of objects in the ocean surface from uavs using a thermal camera. In: IEEE Aerospace Conference, Big Sky (2015)

92. Curtin, T.B., Bellingham, J.G., Catipovic, J., Webb, D.: Autonomous oceanographic sampling networks. Oceanography 6, 86-94 (1993)

93. Leonard, N.E., Paley, D.A., Davis, R.E., Fratantoni, D.M., Lekien, F., Zhang, F.: Coordinated control of an underwater glider fleet in an adaptive ocean sampling field experiment in monterey bay. J. Field Robot. 27, 718-740 (2010)

94. Loy, M., Karingattil, R., Williams, L.: Ism-band and short range device regulatory compliance overview. http://www.ti.com/lit/an/ swra048/swra048.pdf (2005), [Online; Accessed 13 July 2016]

95. Torrieri, D.: Principles of Spread-Spectrum Communication Systems, 2nd edn. Springer (2011)

96. Bekkadal, F.: Future maritime communications technologies. In: OCEANS 2009 - EUROPE, pp. 1-6 (2009)

97. Ge, Y., Kong, P.Y., Tham, C.K., Pathmasuntharam, J.S.: Connectivity and route analysis for a maritime communication network. In: 2007 6th International Conference on Information, Communications Signal Processing, pp. 1-5 (2007)

98. Friderikos, V., Papadaki, K., Dohler, M., Gkelias, A., Agvhami, H.: Linked waters. Commun. Eng. 3(2), 24-27 (2005)

99. Kim, Y., Kim, J., Wang, Y., Chang, K., Park, J.W., Lim, Y.: Application scenarios of nautical ad-hoc network for maritime communications. In: OCEANS 2009, pp. 1-4 (2009)

100. Sozer, E.M., Stojanovic, M., Proakis, J.G.: Underwater acoustic networks. IEEE J. Ocean. Eng. 25(1), 72-83 (2000)

101. Kaushal, H., Kaddoum, G.: Underwater optical wireless communication. IEEE Access 4, 1518-1547 (2016) 
102. Mosca, F., Matte, G., Mignard, V., Rioblanc, M.: Low-frequency source for very long-range underwater communication. In: 2013 OCEANS - San Diego, pp. 1-5 (2013)

103. Stojanovic, M.: Low complexity ofdm detector for underwater acoustic channels. In: OCEANS 2006, pp. 1-6 (2006)

104. Chitre, M., Shahabudeen, S., Freitag, L., Stojanovic, M.: Recent advances in underwater acoustic communications \& networking. In: OCEANS 2008. vol. 2008-Supplement, pp. 1-10 (2008)

105. Stojanovic, M., Catipovic, J., Proakis, J.G.: Adaptive multichannel combining and equalization for underwater acoustic communications. J. Acous. Soc. Amer. 94(3), 1621-1631 (1993). https://doi.org/10.1121/1.408135

106. Murphy, C., Walls, J.M., Schneider, T., Eustice, R.M., Stojanovic, M., Singh, H.: Capture: A communications architecture for progressive transmission via underwater relays with eavesdropping. IEEE J. Ocean. Eng. 39(1), 120-130 (2014)

107. Wang, Y., Liu, Y., Guo, Z.: Three-dimensional ocean sensor networks: A survey. J. Ocean Univ. China 11(4), 436-450 (2012)

108. Pinto, J., Calado, P., Braga, J., Dias, P., Martins, R., Marques, E., Sousa, J.B.: Implementation of a control architecture for networked vehicle systems. In: IFAC Workshop on Navigation, Guidance and Control of Underwater Vehicles (NGCUV) (2012)

109. Mohsin, R.J., Woods, J., Shawkat, M.Q.: Density and mobility impact on manet routing protocols in a maritime environment. In: Science and Information Conference (SAI), 2015, pp. 1046$1051(2015)$

110. Xu, G., Shen, W., Wang, X.: Marine environment monitoring using wireless sensor networks: A systematic review. In: 2014 IEEE International Conference on Systems, Man, and Cybernetics (SMC), pp. 13-18 (2014)

111. Wehs, T., Janssen, M., Koch, C., von Cölln, G.: System architecture for data communication and localization under harsh environmental conditions in maritime automation. In: IEEE 10th International Conference on Industrial Informatics, pp. 1252$1257(2012)$

112. Sanctis, M.D., Cianca, E., Araniti, G., Bisio, I., Prasad, R.: Satellite communications supporting internet of remote things. IEEE Int. Things J. 3(1), 113-123 (2016)

113. Martins, R.: Disruption/delay tolerant networking with lowbandwidth underwater acoustic modems. In: 2010 IEEE/OES Autonomous Underwater Vehicles (2010)

114. Lin, H.M., Ge, Y., Pang, A.C., Pathmasuntharam, J.: Performance study on delay tolerant networks in martitime communication environments. In: IEEE OCEANS (2010)

115. P, S.S., Kumar, S.S.: Sea water quality monitoring using smart sensor network. In: 2015 International Conference on Control, Instrumentation, Communication and Computational Technologies (ICCICCT), pp. 804-812 (2015)

116. Lambrinos, L., Djouvas, C., Chrysostomou, C.: Applying delay tolerant networking routing algorithms in maritime communications. In: 2013 IEEE 14th International Symposium and Workshops on a World of Wireless, Mobile and Multimedia Networks (WoWMoM) (2013)

117. of Technology, M.I.: Moos-ivp home page (2017), http://oceanai. mit.edu/moos-ivp/pmwiki/pmwiki.php

118. Deering, D.S.E.: Internet Protocol, Version 6 (IPv6) Specification. RFC 2460 (2013) https://rfc-editor.org/rfc/rfc2460.txt

119. Luo, H., Wu, K., Guo, Z., Gu, L., Ni, L.M.: Ship detection with wireless sensor networks. IEEE Trans. Parallel Distrib. Syst. 23(7), 1336-1343 (2012)

120. Ho, D.T., Grøtli, E.I., Sujit, P.B., Johansen, T.A., Sousa, J.B.: Optimization of wireless sensor network and uav data acquisition. J. Intell. Robot. Syst. 78(1), 159-179 (2015). https://doi.org/10.1007/s10846-015-0175-5
121. Palattella, M.R., Accettura, N., Vilajosana, X., Watteyne, T., Grieco, L.A., Boggia, G., Dohler, M.: Standardized protocol stack for the internet of (important) things. IEEE Commun. Surv. Tutor. 15(3), 1389-1406 (2013)

122. Palma, D., Curado, M.: Resource Management in Mobile Computing Environments, chap. Scalable Routing Mechanisms for Mobile Ad Hoc Networks, pp. 65-114. Springer International Publishing, Cham (2014)

123. Kim, Y., Kim, J., Wang, Y., Chang, K., Park, J.W., Lim, Y.: Application scenarios of nautical ad-hoc network for maritime communications. In: OCEANS 2009 (2009)

124. Narten, D.T., Jinmei, T., Thomson, D.S.: IPv6 Stateless Address Autoconfiguration. RFC 4862 (2015) https://rfc-editor.org/rfc/ rfc4862.txt

125. Kidston, D., Kunz, T.: Challenges and opportunities in managing maritime networks. IEEE Commun. Mag. 46(10), 162-168 (2008)

126. Costanzo, S., Galluccio, L., Morabito, G., Palazzo, S.: Software Defined Wireless Networks: Unbridling SDNs. In: 2012 European Workshop on Software Defined Networking (EWSDN), pp. 1-6 (2012)

127. Galluccio, L., Milardo, S., Morabito, G., Palazzo, S.: Sdnwise: Design, prototyping and experimentation of a stateful sdn solution for wireless sensor networks. In: 2015 IEEE Conference on Computer Communications (INFOCOM), pp. 513-521 (2015)

Artur Zolich received the MSc degree in Robotics in 2010, from the Wroclaw University of Technology (Wroclaw, Poland). Between 2009 and 2014 he worked on Unmanned Aerial Systems (UAS) development (WB Group, Poland), and aspects of UAS integration into the European Airspace as a member of an ULTRA consortium (Honeywell Aerospace, Czech Republic). Since 2014 he is a PhD candidate at the Department of Engineering Cybernetics, and since 2016 also a Senior Engineer at a Department of Biology at NTNU (Trondheim, Norway). His research focuses on control and communication architectures for coordinated operations of maritime autonomous vehicles and ArcticABC project.

David Palma is an H2020 Marie-Curie Post-Doctoral fellow at the Department of Telematics from NTNU and has worked in the past as an Assistant Professor at the University of Coimbra, as well as a Project Manager at OneSource. He holds a PhD in Information Science and Technology received from the University of Coimbra. His current research interests are on Heterogeneous Networks, Routing and Software-Defined Networking, subjects on which he has authored and co-authored multiple papers in refereed conferences and journals. $\mathrm{He}$ has participated in several TPCs, national and international research projects, including European Projects (FP6/FP7/H2020) and the preparation of successful research proposals.

Kimmo Kansanen received the M.Sc. (EE) and Dr.Tech. degrees from the University of Oulu, Finland, in 1998 and 2005, respectively, where he was a Research Scientist and Project Manager with the Centre for Wireless Communications. Since 2006, he has been with the Norwegian University of Science and Technology, Trondheim, Norway, and as a Professor since 2016. His research interests are within communications and signal processing. He serves on the Editorial Board of Elsevier Physical Communications. 
Kay Fjørtoft finalised his degree at the University of Essex, UK, in 1994, at the department of Computer Science (Artificial Intelligence). He has been a Senior Research Scientist and Research Manager at MARINTEK since 1995, at the department of Maritime Transport Systems, that changed name to SINTEF Ocean in 2017. Current position is a Senior Research Scientist. Kay also held a position at ESA as an Ambassador for the Artes program, where the objectives is to promote space technology in to business applications. Kay is specialised in logistics, maritime communications and integrated operations, and are currently involved in many autonomous vessel projects covering communication and navigation challenges. Kay has published several papers and articles mainly focusing maritime communications, software architecture and logistics challenges, and he is part of the Maritime Communication Center, a program established by the SINTEF Group, the Norwegian University of Science and Technology, and the University of Oslo.

João Sousa is with the Electrical and Computer Engineering Department from Porto University. He is also the head of the Underwater Systems and Technologies Laboratory, an interdisciplinary research laboratory established in 1997 with researchers drawn from Electrical, Computer, and Mechanical Engineering and from Computer Science. His research interests include unmanned vehicles, control architectures, control, and coordination. He has been leading the design, implementation and deployment of advanced unmanned air and ocean vehicle systems in projects funded by the Portuguese Science Foundation (FCT), the Portuguese Ministry of Defence, the Portuguese Innovation Agency (ADI), the European Union (FP6/FP7), NATO and, in the United States of America, the Office of Naval Research and DARPA. In 2006 the laboratory received the national BES Innovation National Award for the design of the Light Autonomous Underwater Vehicle. In 2007 he received an outstanding teaching award from Porto University and has authored over 220 scientific papers.

Karl H. Johansson received the M.Sc. and Ph.D. degrees in electrical engineering from Lund University, Lund, Sweden.He has held visiting positions with the University of California at Berkeley, Berkeley, CA, USA, the California Institute of Technology, Pasadena, CA, USA, Nanyang Technological University, Singapore, and the Institute of Advanced Studies, The Hong Kong University of Science and Technology, Hong Kong. He is currently the Director of the ACCESS Linnaeus Centre and a Professor with the School of Electrical Engineering, KTH Royal Institute of Technology, Stockholm, Sweden, where he heads the Stockholm Strategic Research Area ICT The Next Generation. His current research interests include networked control systems, cyber-physical systems, and applications in transportation, energy, and automation systems.Dr. Johansson is a member of the IEEE Control Systems Society Board of Governors and the European Control Association Council. He received many honours, including Wallenberg Scholar by the Knut and Alice Wallenberg Foundation, Future Research Leader from the Swedish Foundation for Strategic Research, and Young Author Prize from International Federation of Automatic Control.
Yuming Jiang received his Bachelor's degree from Peking University, China, and his Ph.D. degree from National University of Singapore. Since 2005, he has been a professor with the Norwegian University of Science and Technology (NTNU). His main research interest is in the provision and analysis of quality of service guarantees in communication networks. He was General Chair of the IFIP Networking 2014 Conference and is the author of Stochastic Network Calculus.

Hefeng Dong received the B.Sc. and M.Sc. degrees in physics from the Northeast Normal University, Changchun, China, in 1983 and 1986, respectively, and the Ph.D. degree in geoacoustics from the Jilin University, Changchun, China, in 1994.From 1986 to 1994, she was a Lecturer of Physics at the Northeast Normal University, Changchun, China, where she was an Associate Professor from 1995 to 2000. She was a Visiting Scholar and Postdoctoral Fellow at the Norwegian University of Science and Technology, Trondheim, Norway, from 1999 to 2000 and from 2000 to 2001, respectively. From 2001 to 2002, she worked as a Research Scientist at the SINTEF Petroleum Research, Trondheim, Norway. Since 2002, she has been a Professor with the Norwegian University of Science and Technology, Trondheim, Norway. From 2008 to 2009, she was on a one-year sabbatical with the Underwater Acoustics Laboratory, University of Victoria, Victoria, BC, Canada. Her research interests include wave propagation modeling, geoacoustic inversion, and signal processing in ocean acoustics and seismics.Dr. Dong is a member of the Acoustical Society of America, the Norwegian Physical Society, and the Norwegian Acoustical Society.

Tor A. Johansen received the MSc degree in 1989 and the $\mathrm{PhD}$ degree in 1994, both in electrical and computer engineering, from the Norwegian University of Science and Technology, Trondheim, Norway. From 1995 to 1997, he worked at SINTEF as a researcher before he was appointed Associated Professor at the Norwegian University of Science and Technology in Trondheim in 1997 and Professor in 2001. He has published several hundred articles in the areas of control, estimation and optimisation with applications in the marine, automotive, biomedical and process industries. In 2002 Johansen co-founded the company Marine Cybernetics AS where he was Vice President until 2008. Prof. Johansen received the 2006 Arch T. Colwell Merit Award of the SAE, and is currently a principal researcher within the Center of Excellence on Autonomous Marine Operations and Systems (AMOS) and director of the Unmanned Aerial Vehicle Laboratory at NTNU. 